\title{
Herpetofauna de um remanescente de cerrado no estado de São Paulo, sudeste do Brasil
}

\author{
Cybele de Oliveira Araujo ${ }^{1,2,3}$ \& Selma Maria de Almeida-Santos ${ }^{2}$ \\ ${ }^{1}$ Seção de Animais Silvestres, Instituto Florestal, Rua do Horto, 931, \\ Horto Florestal, CEP 02377-000, São Paulo, SP, Brasil \\ ${ }^{2}$ Laboratório de Ecologia e Evolução, Instituto Butantan, \\ Av. Dr. Vital Brazil, 1500, CEP 05503-900, São Paulo, SP, Brasil \\ ${ }^{3}$ Autor para correspondência: Cybele de Oliveira Araujo,e-mail: cyaraujo@usp.br
}

ARAUJO, C.O. \& ALMEIDA-SANTOS, S.M. Herpetofauna in a cerrado remnant in the state of Sao Paulo, Southeastern Brazil. Biota Neotrop. 11(3): http://www.biotaneotropica.org.br/v11n3/en/ abstract?article+bn00511032011

\begin{abstract}
Despite its great extension and biodiversity, there are still sampling gaps in the Cerrado. This region has shown drastic changes due to the conversion of natural areas into pastures and plantations. In the last four decades, around $88.5 \%$ of the Cerrado areas in the state of São Paulo were supressed. Only $0.8 \%$ of Cerrado original cover in the state $(14 \%)$ remain as pristine habitats. We present the species composition, abundance and use of habitat of amphibians and reptiles recorded at Estação Ecológica de Assis, a remnant of Cerrado in the state of São Paulo. The survey was conducted between September 2007 and March 2008 during seven monthly field trips of five days each, totaling 35 sampling days. Surveys were conducted using pitfall traps (AIQ), incidental encounters (EO), auditory encounters (PA), and visual encounters (PV). In addition to the field samples, we included records of species occurring on the municipality of Assis, obtained at the main scientific collections of amphibians and reptiles in the state of São Paulo. We recorded 27 amphibian species, belonging to 13 genera and six families (Bufonidae, Cycloramphidae, Hylidae, Leiuperidae, Leptodactylidae, and Microhylidae), and 53 reptile species belonging to 38 genera and 13 families (Amphisbaenidae, Anguidae, Gekkonidae, Gymnophthalmidae, Polychrotidae, Scincidae, Teiidae, Anomalepididae, Boidae, Colubridae, Dipsadidae, Elapidae, and Viperidae). This survey is an important contribution to the knowledge about these assemblages in the highly threatened Cerrado of the state of São Paulo. Remnants such as the Estação Ecológica de Assis are extremely important for the conservation of amphibians and reptiles in the state and in the Cerrado region.
\end{abstract}

Keywords: amphibians, reptiles, conservation, Cerrado, Assis.

ARAUJO, C.O. \& ALMEIDA-SANTOS, S.M. Herpetofauna de um remanescente de Cerrado no estado de São Paulo, sudeste do Brasil. Biota Neotrop. 11(3): http://www.biotaneotropica.org.br/v11n3/pt/ abstract?article+bn00511032011

Resumo: Apesar da grande extensão e biodiversidade, ainda existem lacunas amostrais no Cerrado. Este domínio fitogeográfico vem sofrendo modificações acentuadas devido à sua conversão em pastagens e monoculturas. Nas últimas quatro décadas ocorreu uma redução de 88,5\% das áreas de Cerrado no estado de São Paulo. Restam menos de $0,8 \%$ da área original do Cerrado paulista, que originalmente cobria $14 \%$ da área do estado. Este estudo apresenta a composição de espécies, abundância e uso de hábitat dos anfíbios e répteis que ocorrem na Estação Ecológica de Assis, um remanescente de Cerrado no estado de São Paulo. O inventário foi realizado entre setembro de 2007 e março de 2008, em sete etapas de campo mensais de cinco dias cada, totalizando 35 dias de trabalho de campo. A amostragem foi realizada por meio de armadilhas de interceptação e queda (AIQ), encontros ocasionais (EO), procura auditiva (PA) e procura visual (PV). Além da amostragem em campo, foram incluídos na lista de espécies os registros obtidos para o município de Assis presentes nas principais coleções científicas de anfíbios e répteis do estado de São Paulo. Foram registradas 27 espécies de anfíbios pertencentes a 13 gêneros e seis famílias (Bufonidae, Cycloramphidae, Hylidae, Leiuperidae, Leptodactylidae e Microhylidae) e 53 espécies de répteis pertencentes a 38 gêneros e 13 famílias (Amphisbaenidae, Anguidae, Gekkonidae, Gymnophthalmidae, Polychrotidae, Scincidae, Teiidae, Anomalepididae, Boidae, Colubridae, Dipsadidae, Elapidae e Viperidae). Este estudo é uma importante contribuição para o conhecimento destas taxocenoses no ameaçado Cerrado paulista. Nesse sentido, remanescentes como a Estação Ecológica de Assis são de extrema importância não só para a conservação da herpetofauna no estado de São Paulo, mas também para o domínio do Cerrado.

Palavras-chave: anfíbios, répteis, conservação, Cerrado, Assis. 


\section{Introdução}

O Brasil é um dos países mais ricos em biodiversidade do planeta, apresentando em seu território dois dos 34 hotspots mundiais prioritários para a conservação biológica, a Mata Atlântica e o Cerrado (Mittermeier et al. 2004). O Cerrado é um dos únicos hotspots em que predomina a vegetação savânica. O seu relevo inclui planaltos, depressões e planícies, cobertos por diferentes fitofisionomias florestais, savânicas e campestres (Fonseca et al. 1999, 2004, Ab'Sáber 2005, Ribeiro \& Walter 2008). A heterogeneidade ambiental deste domínio fitogeográfico está refletida na sua biota que já foi considerada pobre, mas que atualmente passou a ser reconhecida mundialmente pelo elevado grau de endemismo e pressão antrópica a que está sujeita (Colli et al. 2002, Instituto... 2004).

O Cerrado, que possuía originalmente dois milhões de $\mathrm{km}^{2}$ de extensão ( $23 \%$ do território nacional), encontra-se atualmente bastante ameaçado, sendo a agricultura e a pecuária extensiva as responsáveis pela maior parte da perda de áreas de vegetação natural (Ratter et al. 1997, Klink \& Machado 2005, Durigan \& Ratter 2006, Durigan et al. 2007). Apesar de sua grande extensão no território nacional e enorme importância para a conservação da biodiversidade (Myers et al. 2000), apenas 3\% de sua área encontram-se protegidos por unidades de conservação de proteção integral (Brasil \& Instituto... 2009). Estudos recentes estimaram a partir de imagens de satélite que aproximadamente $48 \%$ da cobertura original de Cerrado foram devastados (Brasil \& Instituto... 2009) e se não houver uma reversão nas taxas de ocupação humana, não existirão mais áreas naturais de Cerrado fora das unidades de conservação de proteção integral a partir de 2030 (Machado et al. 2004).

A porção mais ao sul deste domínio, incluindo o Cerrado paulista, é sem dúvida a que se encontra mais severamente impactada. A redução do Cerrado no estado de São Paulo nas últimas quatro décadas foi de $88,5 \%$ da área original, sendo que as culturas que mais contribuíram para a redução deste domínio foram cana-de-açúcar, culturas temporais, citricultura, pastagens e reflorestamento (Kronka et al. 2005). Essa redução resultou em intensa fragmentação, existindo atualmente um total de 7.505 fragmentos, dos quais $71 \%$ são extremamente pequenos (menos de 20 ha), $0,6 \%$ alcançam 400 ha e apenas $0,1 \%$ atingem 10.000 ha (Kronka et al. 2005). Atualmente restam apenas $0,8 \%$ da extensão original de 3,4 milhões ha do Cerrado paulista (14\%) (São Paulo 1997, Kronka et al. 2005). Adicionalmente, somente $0,5 \%$ deste domínio encontram-se protegidos em unidades de conservação no estado e todos esses fragmentos dispersos sofrem ameaças que incluem o isolamento, risco de incêndios e invasão por espécies vegetais exóticas (Durigan et al. 2004, 2007).

Até o momento, são conhecidas para o estado de São Paulo 236 espécies de anfíbios (Araújo et al. 2009b, Rossa-Feres et al. 2011) e 212 espécies de répteis (Zaher et al. 2011), o que representa $27 \%$ e $30 \%$ da riqueza de espécies encontradas no país, respectivamente (Sociedade... 2010, Bérnils 2010). Em sua maior parte, os estudos realizados no estado de São Paulo apresentam informações sobre a diversidade da herpetofauna em localidades de floresta ombrófila presentes no Planalto Atlântico, Serra do Mar e ilhas litorâneas (e.g., Heyer et al. 1990, Marques et al. 2004, 2009, Cicchi et al. 2007, Malagoli 2008, Condez et al. 2009, Narvaes et al. 2009, Araujo et al. 2010a).
Existem inventários de anfíbios em áreas de contato entre formações de Cerrado e floresta estacional nos municípios de Botucatu (Jim 1980, Rossa-Feres \& Jim 1994), Nova Itapirema (Vasconcelos \& Rossa-Feres 2005) e Pedregulho (Araujo et al. 2009a). Em localidades que apresentam predominantemente fitofisionomias de Cerrado há um levantamento de serpentes e lagartos no município de Pirassununga (Vanzolini 1948), inventários de anfíbios (Prado et al. 2009) e serpentes (Dalmolin 2000) na Estação Ecológica de Jataí e região, estudos sobre a diversidade de anfíbios, lagartos e serpentes na Estação Ecológica de Itirapina e entorno (Brasileiro et al. 2005, Thomé 2006, Sawaya et al. 2008), um levantamento sobre as espécies de anfíbios na Estação Ecológica e Floresta Estadual de Assis (Ribeiro-Júnior \& Bertoluci 2009) e dois estudos que incluem a composição de espécies de lagartos (Nogueira et al. 2009) e serpentes (Araujo et al. 2010b) na Estação Ecológica de Santa Bárbara.

Apesar da herpetofauna do estado de São Paulo ser considerada a mais conhecida do país (Rossa-Feres et al. 2011, Zaher et al. 2011), ainda existem áreas não amostradas no território paulista. Estas lacunas de conhecimento encontram-se principalmente em áreas que apresentam remanescentes de Cerrado, como as regiões sudoeste (bacia hidrográfica do rio Paranapanema) e nordeste do estado (Rossa-Feres et al. 2011). Assim, estudos sobre a diversidade da herpetofauna nestas regiões podem gerar informações importantes sobre a biologia e distribuição das espécies, servindo de base para a elaboração de medidas efetivas de manejo e conservação do Cerrado no estado.

O objetivo deste estudo foi caracterizar a herpetofauna da Estação Ecológica de Assis e listar a composição de espécies registrada para o município.

\section{Material e Métodos}

\section{1. Área de estudo}

A Estação Ecológica de Assis (223' $33^{\prime} \mathrm{S}$ e 502 21' O; 500-590 m) localiza-se no município de Assis, estado de São Paulo e apresenta uma área de 1.760,64 ha (Durigan 2010) (Figura 1). O município encontra-se em zona de transição dos tipos climáticos Cwa e Cfa de Köppen (Peel et al. 2007), caracterizados por verões quentes e chuvosos e invernos secos, mas que se diferenciam pela duração do período seco, mais longo ou mais curto, respectivamente (Durigan 2010). A temperatura média anual é de $21,8{ }^{\circ} \mathrm{C}$ e a precipitação pluviométrica anual média varia em torno de $1.450 \mathrm{~mm}$, concentrando-se de outubro a março (Durigan 2010). Geadas fracas ocorrem nas partes mais baixas desta unidade de conservação e geadas muito severas são registradas a cada 25 anos aproximadamente (Durigan 2010). A Estação Ecológica de Assis está inserida na Província Geomorfológica denominada Planalto Ocidental, sendo que no relevo regional predominam declividades baixas (máximo de $15 \%$ ) e amplitudes inferiores a $100 \mathrm{~m}$ (Ponçano et al. 1981). A drenagem é de baixa densidade (padrão sub-dendrítico), com vales abertos e planícies aluviais interiores restritas, com presença eventual de lagoas perenes ou intermitentes (Durigan 2010). Estão inseridas na área as nascentes dos córregos Palmitalzinho, Campestre, Xaxim e Pirapitinga que pertencem às unidades hidrográficas Pari e Capivara (Figura 1), integrantes da bacia do rio Paranapanema (Durigan 2010). A cobertura vegetal predominante nesta estação ecológica é de Cerrado, sendo composta especialmente por formações florestais, como cerradão e mata seca $(67,3 \%)$ e mata de galeria (1,2\%), além de cerrado em regeneração presente em antigos talhões de Eucalyptus sp. 

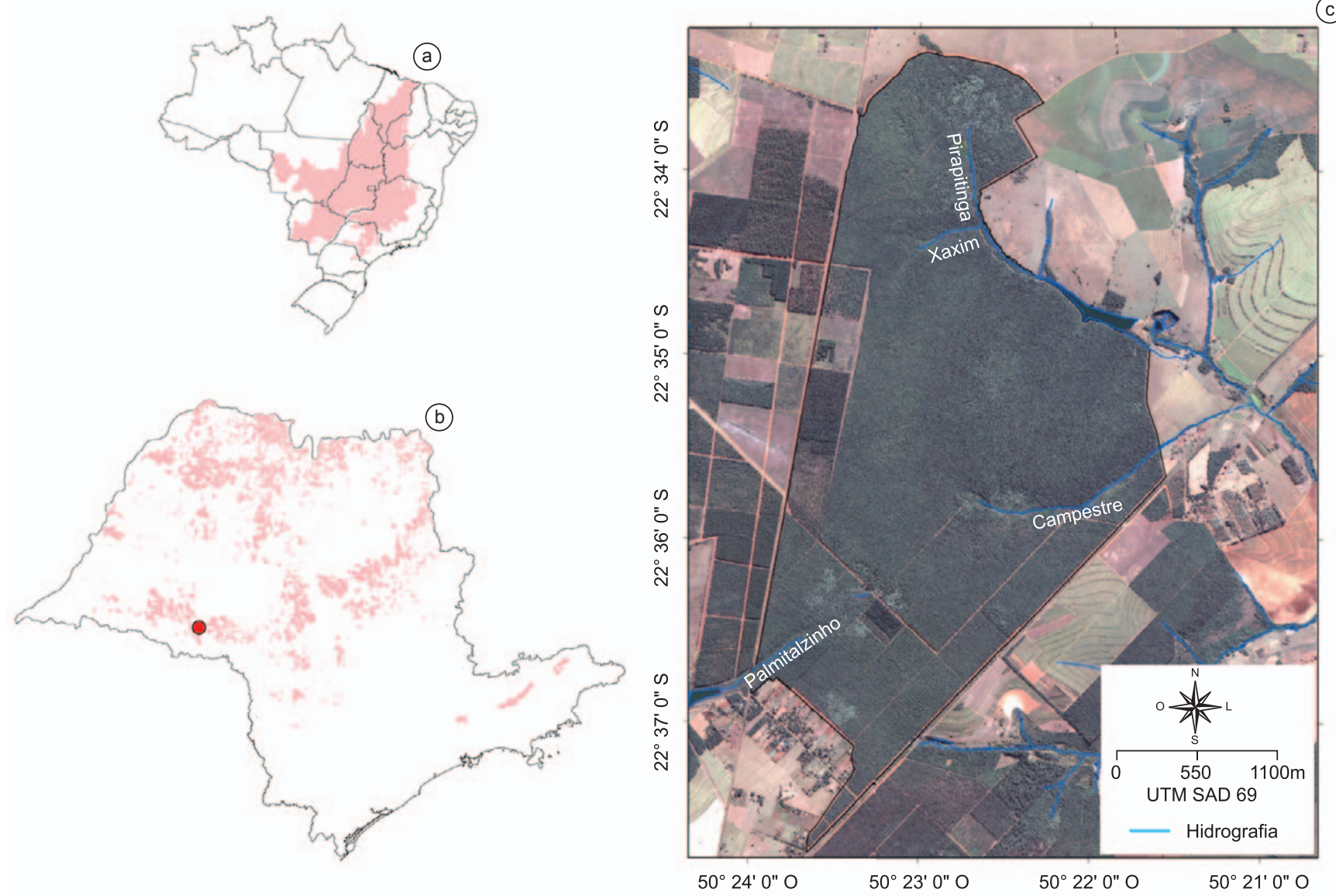

Figura 1. a) Cobertura original do Cerrado no Brasil (fonte: IBGE); b) distribuição original do Cerrado e localização da Estação Ecológica de Assis no estado de São Paulo (fonte: Biota/FAPESP); c) imagem de satélite desta unidade de conservação (fonte: Durigan \& Pinheiro (2010).

Figure 1. a) Original cover of the Cerrado in Brazil (source: IBGE); b) original distribution of the Cerrado and position of the Estação Ecológica de Assis in the state of São Paulo (source: Biota/FAPESP); c) satellite image of this protect area (source: Durigan \& Pinheiro (2010).

(14,9\%) e Pinus sp. (4\%) (Durigan \& Pinheiro 2010). As formações savânicas e campestres, como cerrado denso (1,9\%), cerrado típico $(0,6 \%)$ e campo limpo úmido $(0,3 \%)$, ocupam uma área restrita nesta localidade (Durigan \& Pinheiro 2010) (Figura 2). As fitofisionomias foram classificadas sensu Ribeiro e Walter (2008), na qual o cerrado sentido restrito é subdividido em cerrado denso, cerrado típico e cerrado ralo. Definições sobre as fitofisionomias citadas estão presentes em Durigan et al. (2003) e Ribeiro e Walter (2008).

Segundo análise das primeiras fotografias aéreas disponíveis para a área da Estação Ecológica de Assis (obtidas em 1962), constatou-se que no passado o cerradão ocupava cerca de metade da área, ocorrendo grandes trechos de cerrado sentido restrito (Pinheiro 2008). Uma vez protegida contra incêndios, a vegetação vem passando por um processo contínuo de adensamento, com aumento de cobertura e biomassa (Pinheiro 2008). Este processo está resultando em redução da área das fisionomias campestres e savânicas, que pode acarretar em diminuição da diversidade de espécies vegetais e animais restritas às formações abertas de Cerrado (Durigan \& Pinheiro 2010).

\section{Coleta e análise dos dados}

O inventário da herpetofauna foi realizado entre setembro de 2007 e março de 2008, em sete etapas de campo mensais de cinco dias cada, totalizando 35 dias de amostragem. Foram utilizados três métodos complementares de amostragem ativa: procura auditiva, procura visual e encontro ocasional (Crump \& Scott Junior 1994, Sawaya 2004). A procura auditiva (PA) e visual (PV) consistiu em censos realizados no período noturno por duas pessoas, em transectos previamente definidos. Foram examinados os microhábitats acessíveis (e.g., bromélias, folhiço) e os sítios reprodutivos utilizados pelos anuros (e.g., brejos, poças permanentes e temporárias, riachos, várzeas). A duração da amostragem variou entre quatro e seis horas por dia, resultando em um esforço total de aproximadamente 130 horas de procura e esforço empregado de 65 horas-homem. Foram amostrados sete ambientes aquáticos utilizados principalmente pelos anuros: brejo em cerrado típico, uma lagoa e dois riachos em mata de galeria, poça temporária em aceiro, poça temporária na beira de estrada e represa (Figura 2; Tabela 1). O método de encontro ocasional (EO) consistiu no registro de espécimes encontrados vivos ou mortos, quando não estava sendo realizado nenhum tipo de procura. Embora o esforço de amostragem não seja considerado, este método é muito útil na obtenção de animais para o registro da riqueza, produzindo listas mais completas e informações sobre o comportamento das espécies (e.g., uso de hábitat, dieta, atividade reprodutiva) (Martins 1994, Sawaya 2004). 
Também foram realizadas amostragens por meio de armadilhas de interceptação e queda (pitfall traps), interligadas por cercas-guia (drift-fences) (Corn 1994, Cechin \& Martins 2000). Com o objetivo de caracterizar o uso de hábitat e determinar a abundância dos anfíbios e répteis nas diferentes fitofisionomias da Estação Ecológica de Assis, foram instaladas duas linhas com quatro baldes plásticos de $60 \mathrm{~L}$ e espaçadas em $100 \mathrm{~m}$ em cada uma das seis áreas selecionadas. As doze linhas, em um total de 48 armadilhas (esforço amostral $=1.680 \mathrm{baldes} / \mathrm{dia}$ ), foram distribuídas em quatro diferentes fitofisionomias: seis linhas em cerradão, duas linhas em cerrado típico, duas linhas em mata de galeria e duas linhas em área de contato de cerradão e mata de galeria (Figura 2; Tabela 1).

A eficiência da amostragem foi avaliada por meio de curvas de rarefação (Gotelli \& Colwell 2001) construídas com dados de presença e ausência das espécies na localidade nos 35 dias de amostragem (uma amostra $=$ um dia). A riqueza de espécies foi estimada utilizando os algoritmos Bootstrap e Jacknife de primeira ordem (Jacknife I). Para a escolha dos estimadores de riqueza, foram realizadas comparações visuais do desempenho de curvas de rarefação de sete estimadores: ACE e ICE; Chao 1 e 2; Jacknife I e II; Bootstrap (Moraes et al. 2007, Ribeiro-Júnior \& Bertoluci 2009, Brassaloti et al. 2010). As curvas foram geradas pelo Programa Estimates com 10.000 aleatorizações (Colwell 2009) (Figura 3).

Com o intuito de determinar a riqueza de espécies da herpetofauna presente nesta região (município de Assis), além da amostragem realizada na Estação Ecológica de Assis, foram incluídos neste estudo os registros de tombo obtidos nas principais coleções

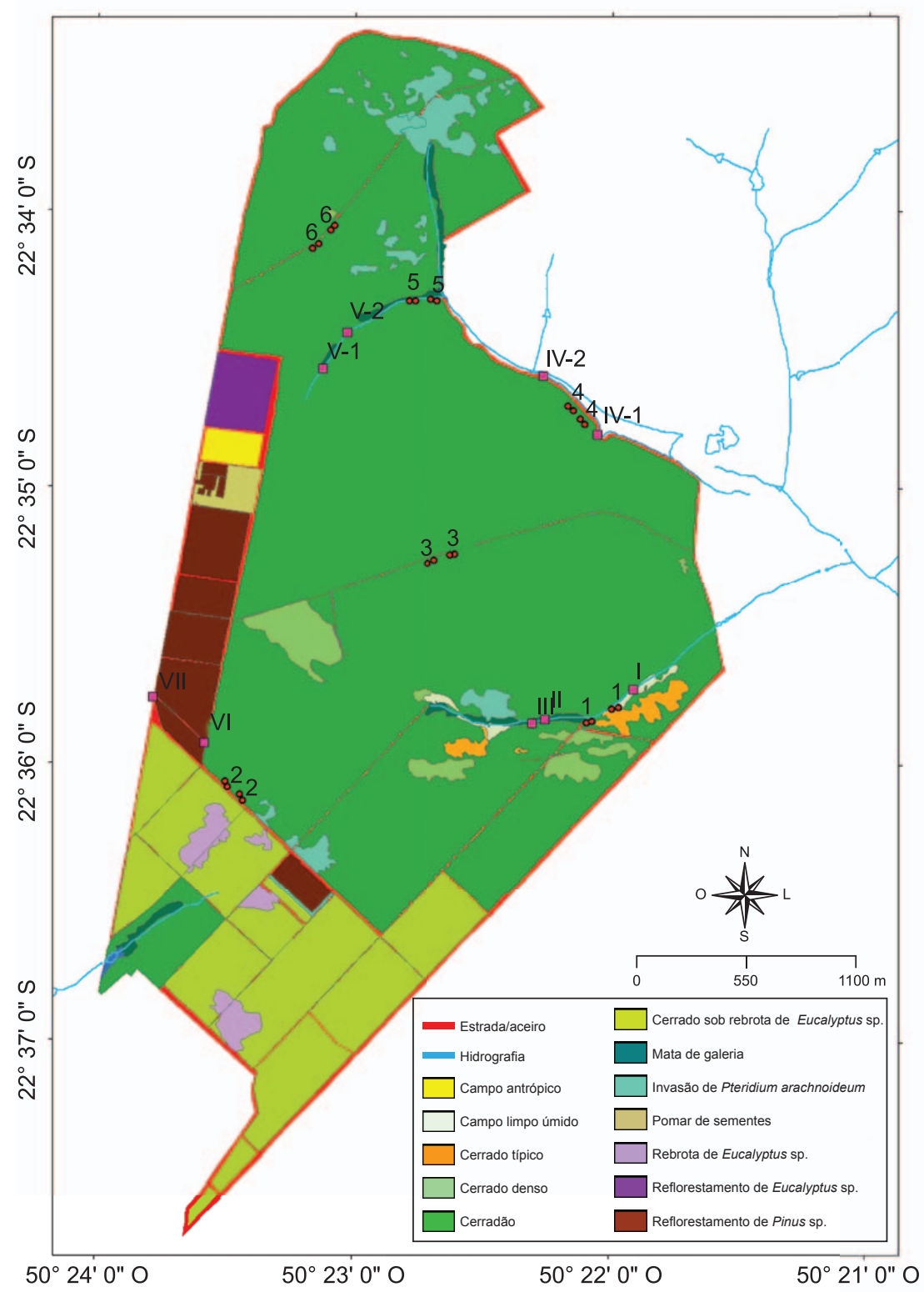

Figura 2. Fitofisionomias da Estação Ecológica de Assis, SP e áreas de amostragem (pontos em vermelho = linhas de armadilhas de interceptação e queda; pontos em rosa = ambientes aquáticos). Fonte: modificado de Durigan \& Pinheiro (2010).

Figure 2. Phytophysiognomies of the Estação Ecológica de Assis, state of São Paulo, Brazil and sampled areas (red dots = pitfall traps lines; pink dots = aquatic sites). Source: modified from Durigan \& Pinheiro (2010). 
científicas de anfíbios e répteis do estado de São Paulo: Coleção de Anfíbios do Departamento de Zoologia da Universidade Estadual Paulista "Júlio de Mesquita Filho", campus de Rio Claro (CFBH), Coleção de Anfíbios da Universidade Estadual Paulista "Júlio de Mesquita Filho", campus de São José do Rio Preto (DZSJRP), Coleção Herpetológica "Alphonse Richard Hoge" do Instituto Butantan (IBSP), Coleção Herpetológica do Museu de Zoologia da Universidade de São Paulo (MZUSP) e Coleção Herpetológica do Museu de História Natural, Universidade Estadual de Campinas (ZUEC) (Tabela 2). Não foram encontrados registros de espécies para o município de Assis nas coleções de anfíbios CFBH e DZSJRP. Apenas as espécies taxonomicamente determinadas ou registradas como "cf." (confer) foram incorporadas à listagem desta região. A nomenclatura utilizada segue as propostas taxonômicas mais recentes para os anfíbios (Faivovich et al. 2005, Frost et al. 2006, Grant et al. 2006, Nascimento et al. 2006, Aguiar Junior et al. 2007, Chaparro et al. 2007, Frost 2011, Lavilla et al. 2010, Toledo et al. 2010, Rossa-Feres et al. 2011) e répteis (Frost et al. 2001, Curcio et al. 2009, Fenwick et al. 2009, Mott \& Vieites 2009, Zaher et al. 2009, Bisby et al. 2010, Zaher et al. 2011).

O status de conservação de cada espécie foi obtido na lista vermelha internacional de espécies ameaçadas de extinção da União Internacional para Conservação da Natureza (International... 2010), lista nacional das espécies da fauna brasileira ameaçadas de extinção (Instituto... 2003) e lista de anfíbios e répteis ameaçados do estado de São Paulo (São Paulo 2008) (Tabela 2).

Os espécimes coletados (licenças de coleta IBAMA/RAN 10423-1 e 13706-1) foram depositados nas coleções CFBH (anfíbios), IBSP (serpentes e lagartos) e MZUSP (lagartos) (Apêndice 1).

\section{Resultados e Discussão}

\section{Composição de espécies}

Registrou-se na Estação Ecológica de Assis e município de Assis um total de 27 espécies de anfíbios e 53 répteis, o que demonstra uma alta riqueza de espécies da herpetofauna para esta região. Foram amostradas seis famílias de anfíbios anuros: Bufonidae (1 espécie), Cycloramphidae (1), Hylidae (12), Leiuperidae (5), Leptodactylidae (6) e Microhylidae (2) (Tabela 2). Em relação aos répteis, foram registradas quatro espécies de anfisbenídeos (Amphisbaenidae) e seis famílias de lagartos: Anguidae (1 espécie), Gekkonidae (1), Gymnophthalmidae (2), Polychrotidae (2), Scincidae (1) e Teiidae (3). As serpentes encontradas pertencem a seis famílias: Anomalepididae (1 espécie), Boidae (2), Colubridae (4), Dipsadidae (25), Elapidae (2) e Viperidae (5) (Tabela 3).

Durante a amostragem de campo foram registradas 22 espécies de anfíbios e 23 répteis (Figuras 4 e 5). Deste total, duas espécies de anfíbios anuros (Hypsiboas caingua e Scinax similis), oito lagartos (Ameiva ameiva, Anolis chrysolepis, Cnemidophorus gr. ocellifer, Colobosaura modesta, Mabuya frenata, Micrablepharus atticolus, Ophiodes sp. e Tupinambis merianae) e uma serpente (Phalotris lativittatus) representam novos registros para o município de Assis (Tabelas 2 e 3).

\section{Diversidade e uso de hábitat das espécies}

Dos anuros amostrados neste estudo (22 espécies), apenas três foram observadas exclusivamente em formações florestais durante a atividade reprodutiva. Hypsiboas caingua e Hypsiboas lundii foram encontradas vocalizando na mata de galeria ao longo de pequenos riachos durante todo o período de amostragem, enquanto Phyllomedusa tetraploidea foi observada vocalizando na borda do cerradão, próxima a uma poça temporária formada com as chuvas de verão (dezembro a fevereiro) (Tabelas 1 e 2). Treze espécies foram observadas em atividade reprodutiva exclusivamente em áreas com formações abertas (brejo em cerrado típico, poça temporária em área alterada e represa): Dendropsophus nanus, Elachistocleis bicolor, Eupemphix nattereri, Leptodactylus furnarius, L. fuscus, L. labyrinthicus, L. latrans, L. mystacinus, L. podicipinus, Physalaemus marmoratus, Scinax fuscomarginatus, S. fuscovarius e $S$. similis (Tabelas 1 e 2). Cinco espécies (Rhinella schneideri,

Tabela 1. Linhas de armadilhas de interceptação e queda e ambientes aquáticos amostrados na Estação Ecológica de Assis, SP. Fitofisionomias (área alterada, campo limpo úmido, cerradão, cerrado típico, mata de galeria), tipo de hábitat (aberto, florestal) e coordenadas geográficas.

Table 1. Pitfall traps lines and aquatic sites sampled at the Estação Ecológica de Assis, state of São Paulo, Brazil. Phytophysiognomies (campo limpo úmido, cerradão, cerrado típico, gallery forest, modified area), type of environment (open, forest) and geographic coordenates.

\begin{tabular}{|c|c|c|c|}
\hline $\begin{array}{c}\text { Linhas de armadilhas }\left(\mathrm{n}^{\circ}\right) / \\
\text { sítio aquático }\left(\mathrm{n}^{\circ}\right)\end{array}$ & Fitofisionomia & $\begin{array}{l}\text { Tipo de } \\
\text { hábitat }\end{array}$ & $\begin{array}{c}\text { Coordenada } \\
\text { geográfica }\end{array}$ \\
\hline 1 & Cerrado típico & Aberto & $22^{\circ} 35^{\prime} 48^{\prime \prime}-51^{\prime \prime} \mathrm{S}$ e $50^{\circ} 22^{\prime} 00^{\prime \prime}-06^{\prime \prime} \mathrm{O}$ \\
\hline 2 & Cerradão próx. talhão Eucaliptus sp. & Florestal & $22^{\circ} 36^{\prime} 4 ”-8 ” \mathrm{~S}$ e $50^{\circ} 23^{\prime} 26^{\prime \prime}-30^{\prime \prime} \mathrm{O}$ \\
\hline 3 & Cerradão & Florestal & $22^{\circ} 35^{\prime} 15^{\prime \prime}-16^{\prime \prime} \mathrm{S}$ e $50^{\circ} 22^{\prime} 36^{\prime \prime}-43^{\prime \prime} \mathrm{O}$ \\
\hline 4 & Cerradão/matade galeria & Florestal & $22^{\circ} 34^{\prime} 42^{\prime \prime}-46^{\prime \prime} \mathrm{S}$ e $50^{\circ} 22^{\prime} 06^{\prime \prime}-10^{\prime \prime} \mathrm{O}$ \\
\hline 5 & Mata de galeria & Florestal & $22^{\circ} 34^{\prime} 20^{\prime \prime}-21^{\prime \prime}$ S e $50^{\circ} 22^{\prime} 41^{\prime \prime}-47^{\prime \prime} \mathrm{O}$ \\
\hline 6 & Cerradão em regeneração & Florestal & $22^{\circ} 34^{\prime} 3 ”-8 ” \mathrm{~S}$ e $50^{\circ} 233^{\prime} 5^{\prime \prime}-10^{\prime \prime} \mathrm{O}$ \\
\hline Brejo (I) & Cerrado típico & Aberto & $22^{\circ} 35^{\prime} 44^{\prime \prime} \mathrm{S}$ e $50^{\circ} 21^{\prime} 55^{\prime \prime} \mathrm{O}$ \\
\hline Riacho (II) & Mata de galeria & Florestal & $22^{\circ} 35^{\prime} 50^{\prime \prime} \mathrm{S}$ e $50^{\circ} 22^{\prime} 15^{\prime \prime} \mathrm{O}$ \\
\hline Lagoa (III) & Mata de galeria & Florestal & $22^{\circ} 35^{\prime} 51^{\prime \prime} \mathrm{S}$ e $50^{\circ} 22^{\prime} 18^{\prime \prime} \mathrm{O}$ \\
\hline Represa (IV-1/IV-2 ) & Campo limpo úmido & Aberto & $22^{\circ} 34^{\prime} 36^{\prime \prime}-47^{\prime \prime} \mathrm{S}$ e $50^{\circ} 22^{\prime} 3 "-16^{\prime \prime} \mathrm{O}$ \\
\hline Riacho (V-1/V-2) & Mata de galeria & Florestal & $22^{\circ} 34^{\prime} 20^{\prime \prime}-14^{\prime \prime} \mathrm{S}$ e $50^{\circ} 22^{\prime} 40^{\prime \prime}-48^{\prime \prime} \mathrm{O}$ \\
\hline Poça temporária - aceiro (VI) & Área alterada & Aberto & $22^{\circ} 35^{\prime} 56^{\prime \prime} \mathrm{S}$ e $50^{\circ} 23^{\prime} 35^{\prime \prime} \mathrm{O}$ \\
\hline Poça temporária - estrada (VII) & Área alterada & Aberto & $22^{\circ} 35^{\prime} 46^{\prime \prime} \mathrm{S}$ e $50^{\circ} 23^{\prime} 47^{\prime \prime} \mathrm{O}$ \\
\hline
\end{tabular}



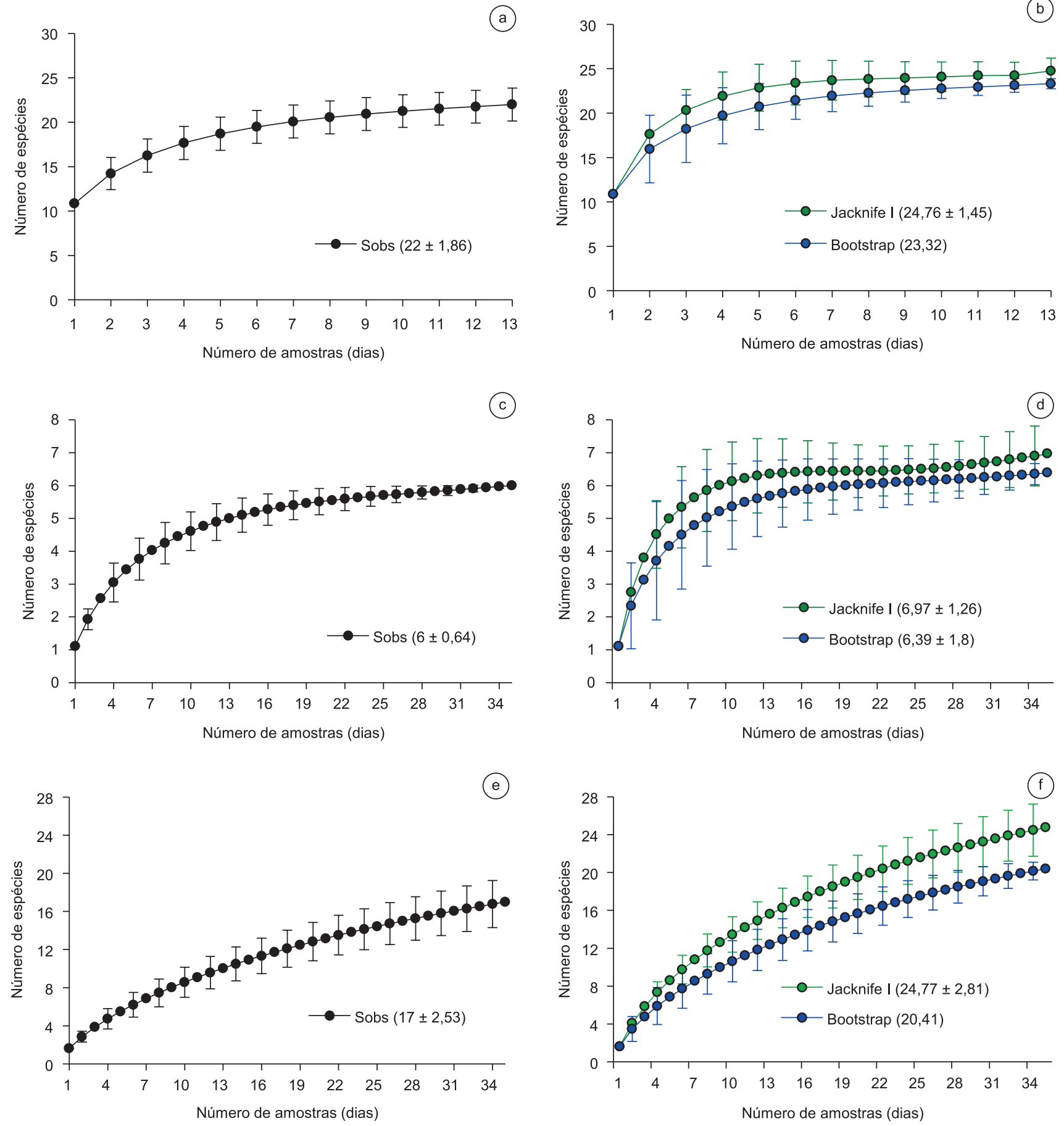

Figura 3. Curvas de rarefação representando as riquezas de espécies observadas (linhas pretas) e curvas de riquezas estimadas geradas pelos estimadores Bootstrap (linhas azuis) e Jacknife I (linhas verdes) para 13 (PA; PV) e 35 (AIQ) dias de amostragem (setembro de 2007 a março de 2008 ) na Estação Ecológica de Assis, SP. Métodos de amostragem: a; b) procura auditiva (PA) e visual (PV) para os anfíbios; c; d) armadilhas de interceptação e queda (AIQ) para os anfíbios; e; f) para os répteis. As barras verticais correspondem aos desvios padrão das riquezas de espécies observadas e riquezas estimadas. Curvas geradas com 10.000 aleatorizações (Colwell 2009).

Figure 3. Rarefaction curves representing observed species richness (black lines) and estimated richness curves generated by the estimators Bootstrap (blue lines) and Jacknife I (green lines) to 13 (PA; PV) and 35 (AIQ) sampled days (September 2007 to March 2008) at the Estação Ecológica de Assis, state of São Paulo. Methods of sampling: a; b) auditory encounters (PA) and visual encounters (PV) to amphibians; c; d) pitfall traps (AIQ) to amphibians; e; f) reptiles. Vertical bars correspond to standard deviations of observed species richness and estimated richness. Curves were created by means of 10,000 randomizations (Colwell 2009). 
Dendropsophus minutus, Hypsiboas albopunctatus, H. faber e Physalaemus cuvieri) foram encontradas em sítios reprodutivos presentes em formações abertas e florestais. A espécie Chiasmocleis albopunctata não foi encontrada em atividade de vocalização durante a amostragem (Tabelas 1 e 2). A maior riqueza de espécies de anfíbios anuros em formações abertas já foi demonstrada em outros estudos que compararam a riqueza de espécies em formações campestres, savânicas e florestais em regiões de Cerrado (e.g., Brandão \& Araújo 2002, Brasileiro et al. 2005, Thomé 2006, Araujo et al. 2009a).

Nas armadilhas de interceptação e queda (AIQ) foram capturadas seis espécies de anuros $(\mathrm{N}=141)$, sete lagartos $(\mathrm{N}=59)$, oito serpentes $(\mathrm{N}=11)$ e dois anfisbenídeos $(\mathrm{N}=3)$ (Figura 6).

Considerando apenas as capturas efetuadas nas AIQ, as espécies mais abundantes de anfíbios foram Physalaemus cuvieri $(\mathrm{N}=85$; $60,3 \%)$, Eupemphixnattereri $(\mathrm{N}=31 ; 22 \%)$ e Leptodactylus mystacinus

Tabela 2. Anfíbios da Estação Ecológica de Assis e município de Assis, SP (DP = dados primários, LC = literatura e DS = dados secundários). Coleções científicas: $\mathrm{M}=$ Coleção Herpetológica do Museu de Zoologia da Universidade de São Paulo (MZUSP) e Z = Coleção Herpetológica do Museu de História Natural, Universidade Estadual de Campinas (ZUEC). Métodos de amostragem: AIQ = armadilhas de interceptação e queda, PA = procura auditiva e PV = procura visual. Locais de captura: linhas de armadilhas e ambientes aquáticos.

Table 2. Amphibians from Estação Ecológica de Assis and municipality of Assis, SP (DP = primary data, LC = literature, and DS = secondary data). Scientific collections: M = Coleção Herpetológica do Museu de Zoologia da Universidade de São Paulo (MZUSP) and Z = Coleção Herpetológica do Museu de História Natural, Universidade Estadual de Campinas (ZUEC). Methods of sampling: AIQ = pitfall traps, PA = auditory encounters, and PV = visual encounters. Local of capture: pitfall traps lines and aquatic sites.

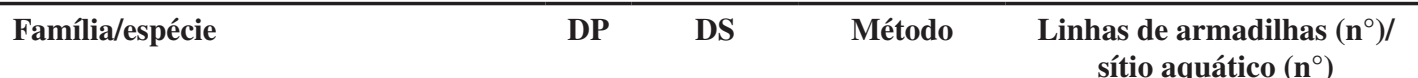

Bufonidae

Rhinella schneideri (Werner, 1894)

Cycloramphidae

Odontophrynus americanus (Duméril \& Bibron, 1841)

Hylidae

Dendropsophus elianeae (Napoli \& Caramaschi, 2000)

Dendropsophus minutus (Peters, 1872)

Dendropsophus nanus (Boulenger, 1889)

Hypsiboas albopunctatus (Spix, 1824)

Hypsiboas caingua (Carrizo, 1991 “1990”)

Hypsiboas faber (Wied-Neuwied, 1821)

Hypsiboas lundii (Burmeister, 1856)

Phyllomedusa tetraploidea Pombal \& Haddad, 1992

Pseudis platensis Gallardo, 1961

Scinax fuscomarginatus (A. Lutz, 1925)

Scinax fuscovarius (A. Lutz, 1925)

Scinax similis (Cochran, 1952)

Leiuperidae

Eupemphix nattereri Steindachner, 1863

Physalaemus centralis Bokermann, 1962

Physalaemus cuvieri Fitzinger, 1826

Physalaemus marmoratus (Reinhardt \& Lütken, 1862 "1861”)

Pseudopaludicola cf. mystacalis (Cope 1887)

Leptodactylidae

Leptodactylus furnarius Sazima \& Bokermann, 1978

Leptodactylus fuscus (Schneider, 1799)

Leptodactylus labyrinthicus (Spix, 1824)

Leptodactylus latrans (Steffen, 1815)

Leptodactylus mystacinus (Burmeister, 1861)

Leptodactylus podicipinus (Cope, 1862)

Microhylidae

Chiasmocleis albopunctata (Boettger, 1885)

Elachistocleis bicolor (Valenciennes in Guérin-Menéville, 1838)

\begin{tabular}{|c|c|c|c|}
\hline • & $\mathrm{LC}$ & AIQ; PA; PV & 5/III; IV \\
\hline- & $\mathrm{LC} ; \mathrm{M}$ & - & - \\
\hline- & M & - & - \\
\hline$\bullet$ & $\mathrm{LC}$ & PA; PV & I; III; IV; VII \\
\hline$\bullet$ & $\mathrm{LC}$ & PA; PV & I; IV; VII \\
\hline$\bullet$ & $\mathrm{LC}$ & PA; PV & I; II; III; IV; V \\
\hline$\bullet$ & & PA; PV & I; IV; V \\
\hline$\bullet$ & LC; M; Z & PA; PV & I; II; III; IV; V \\
\hline$\bullet$ & LC & PA; PV & I; II; III; IV; V \\
\hline$\bullet$ & $\mathrm{LC} ; \mathrm{Z}$ & PA; PV & VI; VII \\
\hline- & M & - & - \\
\hline$\bullet$ & $\mathrm{LC}$ & PA; PV & I; IV \\
\hline$\bullet$ & LC; M & PA; PV & VI; VII \\
\hline$\bullet$ & & PA; PV & I; IV \\
\hline$\bullet$ & $\mathrm{LC}$ & AIQ; PA; PV & $1 ; 2 ; 3 ; 4 ; 5 ; 6 / \mathrm{I} ; \mathrm{VI} ; \mathrm{VII}$ \\
\hline- & M & - & - \\
\hline$\bullet$ & $\mathrm{LC} ; \mathrm{M}$ & AIQ; PA; PV & 1; 4; 5; 6/I; II; III; IV; V; VII \\
\hline$\bullet$ & $\mathrm{LC} ; \mathrm{M}$ & $\mathrm{PA} ; \mathrm{PV}$ & VII \\
\hline- & $\mathrm{LC}$ & - & - \\
\hline$\bullet$ & $\mathrm{LC}$ & PA; PV & I \\
\hline$\bullet$ & $\mathrm{LC} ; \mathrm{M}$ & PA; PV & IV; VI; VII \\
\hline$\bullet$ & LC & PA; PV & I; IV; VII \\
\hline$\bullet$ & $\mathrm{LC}$ & PA; PV & IV \\
\hline • & $\mathrm{LC}$ & AIQ; PA; PV & $1 ; 2 ; 3 ; 4 ; 5 ; 6 / \mathrm{IV} ; \mathrm{VII}$ \\
\hline$\bullet$ & $\mathrm{LC}$ & PA; PV & IV \\
\hline 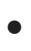 & $\mathrm{LC}$ & AIQ & $1 ; 2 ; 4 ; 5$ \\
\hline$\bullet$ & - & AIQ; PA; PV & 1; 2; 4/I; IV; VII \\
\hline
\end{tabular}


( $\mathrm{N}=10 ; 7,1 \%$ ), que estavam amplamente distribuídas nas formações abertas e florestais da Estação Ecológica de Assis (Figura 6; Tabelas 1 e 2). A espécie dominante na formação aberta (cerrado típico) foi E. nattereri $(\mathrm{N}=10 ; 52,6 \%)$. Já nas formações florestais (cerradão e mata de galeria), a espécie mais frequente foi $P$. cuvieri $(\mathrm{N}=80 ; 65 \%)$. Estudos realizados na Estação Ecológica de Itirapina apontaram $P$. cuvieri, $P$. centralis $(43,6 \%)$, consideradas em conjunto pela dificuldade na identificação dos juvenis e P. marmoratus $(31,9 \%)$ como as espécies mais abundantes nas formações abertas (Brasileiro 2004) e Rhinella ornata (95\%) nas fisionomias florestais, como o cerradão (Thomé 2006). Embora a fisionomia vegetal predominante na Estação Ecológica de Assis seja o cerradão e seu ecótono com mata seca $(67,3 \%)$, a espécie $R$. ornata não foi encontrada nesta unidade de conservação.

Entre os répteis, as espécies mais frequentes foram os lagartos Ameiva ameiva $(\mathrm{N}=31 ; 42,5 \%)$, observado quase que exclusivamente em cerrado típico $(\mathrm{N}=30)$, Colobosaura modesta $(\mathrm{N}=9 ; 12,3 \%)$ e Mabuya frenata $(\mathrm{N}=9 ; 12,3 \%)$, amostrados apenas nas formações florestais (Figura 6; Tabelas 1 e 3). De forma diferente, os lagartos mais abundantes na Estação Ecológica de Itirapina foram Cnemidophorus cf. mumbuca (30,8\%), Kentropyx paulensis $(26,6 \%)$ e Cercosaura schreibersii (14,8\%), espécies típicas de fisionomias abertas (Thomé 2006), formações vegetais estas com baixa representatividade na Estação Ecológica de Assis. Ameiva ameiva também foi a espécie mais frequente entre os lagartos no cerrado sentido restrito da Estação Ecológica de Itirapina, porém apresentou uma menor dominância (53\%) em relação à encontrada na Estação Ecológica de Assis (85,7\%).

Dentre as espécies registradas, 16 anuros, dois lagartos e três serpentes foram amostradas exclusivamente por meio de procura auditiva (anfíbios), procura visual e encontro ocasional (Tabelas 2 e 3). Para os anfíbios, os métodos de amostragem ativa (PA, PV e EO) contribuíram mais efetivamente para este inventário.
Já para os anfisbenídeos e lagartos, o método de amostragem passiva (AIQ) foi o mais relevante. Desta forma, é importante ressaltar a necessidade da utilização de métodos complementares (amostragem ativa e passiva) para a obtenção de listas mais completas. As serpentes são relativamente difíceis de serem amostradas e a combinação de diferentes métodos de amostragem se faz necessária pelas limitações inerentes a cada método (Sawaya 2004). Neste caso, a utilização de dados secundários (coleções científicas) foi imprescindível para gerar uma lista de espécies mais abrangente (Tabela 3).

Foram feitas curvas de rarefação da riqueza de espécies observadas e estimadas (estimadores Bootstrap e Jacknife I), para a procura auditiva (PA) e visual (PV), em 13 dias de amostragem e para armadilhas de interceptação e queda (AIQ), em 35 dias de amostragem (Figura 3). Devido à baixa taxa de captura de répteis utilizando os métodos de procura ativa, não foi possível construir curvas de rarefação.

As curvas de rarefação da riqueza observada e estimada para anfíbios por procura ativa (PA e PV) (Figuras 3a,b) e por AIQ (Figuras 3c,d) apresentaram tendência à estabilização, indicando que, possivelmente, boa parte das espécies foi amostrada durante o estudo. Os estimadores utilizados indicaram que podem ocorrer até cinco espécies a mais por procura ativa (riqueza observada $=$ $22 \pm 1,86$; Bootstrap $=23,32$; Jacknife $\mathrm{I}=24,76 \pm 1,45$ ) e duas ou três espécies a mais por AIQ (riqueza observada $=6 \pm 0,64$; Bootstrap $=6,39 \pm 1,8$; Jacknife $\mathrm{I}=6,97 \pm 1,26$ ). Estas estimativas são corroboradas por Ribeiro-Júnior e Bertoluci (2009), que indicaram a presença de mais duas espécies para a Estação Ecológica de Assis (Odontophrynus americanus e Pseudopaludicola cf. mystacalis) e pelos registros de outras três espécies para o município de Assis (Dendropsophus elianeae, Physalaemus centralis e Pseudis platensis) (Tabela 3).

Para os répteis, as curvas de rarefação da riqueza observada e estimada para as espécies amostradas por AIQ (Figuras 3e, f) não

Tabela 3. Répteis da Estação Ecológica de Assis e município de Assis, SP (DP = dados primários e DS = dados secundários). Coleções científicas: $\mathrm{I}=$ Coleção Herpetológica "Alphonse Richard Hoge" do Instituto Butantan (IBSP), M = Coleção Herpetológica do Museu de Zoologia da Universidade de São Paulo (MZUSP) e Z = Coleção Herpetológica do Museu de História Natural, Universidade Estadual de Campinas (ZUEC). Métodos de amostragem: AIQ = armadilhas de interceptação e queda, EO = encontro ocasional e PV = procura visual. Locais de captura: linhas de armadilhas e ambientes aquáticos.

Table 3. Reptiles from Estação Ecológica de Assis and municipality of Assis, SP (DP = primary data and DS = secondary data). Scientific collections: $\mathrm{I}=$ Coleção Herpetológica "Alphonse Richard Hoge" do Instituto Butantan (IBSP), M = Coleção Herpetológica do Museu de Zoologia da Universidade de São Paulo (MZUSP) and Z = Coleção Herpetológica do Museu de História Natural, Universidade Estadual de Campinas (ZUEC). Methods of sampling: AIQ = pitfall traps, $\mathrm{EO}=$ incidental encounters, and PV = visual encounters. Local of capture: pitfall traps lines and aquatic sites.

\begin{tabular}{|c|c|c|c|c|}
\hline Família/espécie & DP & DS & Método & $\begin{array}{c}\text { Linhas de armadilhas }\left(\mathrm{n}^{\circ}\right) / \\
\text { sítio aquático }\left(\mathrm{n}^{\circ}\right)\end{array}$ \\
\hline \multicolumn{5}{|l|}{ Amphisbaenidae } \\
\hline Amphisbaena alba Linnaeus, 1758 & $\bullet$ & M & AIQ & $5 ; 6$ \\
\hline Amphisbaena mertensii Strauch, 1881 & - & M & - & - \\
\hline Amphisbaena microcephala (Wagler, 1824) & - & M & - & - \\
\hline Amphisbaena roberti Gans, 1964 & $\bullet$ & M & AIQ & 3 \\
\hline \multicolumn{5}{|l|}{ Anguidae } \\
\hline Ophiodes sp. & $\bullet$ & - & $\mathrm{EO}$ & próx. VI \\
\hline \multicolumn{5}{|l|}{ Gekkonidae } \\
\hline Hemidactylus mabouia (Moreau de Jonnès, 1818) & & M & - & - \\
\hline \multicolumn{5}{|l|}{ Gymnophthalmidae } \\
\hline Colobosaura modesta (Reinhardt \& Luetken, 1862) & $\bullet$ & - & AIQ & $4 ; 5 ; 6$ \\
\hline Micrablepharus atticolus Rodrigues, $1996^{1}$ & $\bullet$ & - & AIQ & 1 \\
\hline
\end{tabular}

'Espécie vulnerável (VU) segundo a lista de espécies ameaçadas do estado de São Paulo (São Paulo 2008). 
Tabela 3. Continuação...

\begin{tabular}{|c|c|c|c|c|}
\hline Família/espécie & DP & DS & Método & $\begin{array}{c}\text { Linhas de armadilhas }\left(\mathrm{n}^{\circ}\right) / \\
\text { sítio aquático }\left(\mathrm{n}^{\circ}\right)\end{array}$ \\
\hline \multicolumn{5}{|l|}{ Polychrotidae } \\
\hline Anolis chrysolepis Duméril \& Bibron, 1837 & $\bullet$ & - & AIQ & $2 ; 4 ; 5$ \\
\hline Polychrus acutirostris Spix, 1825 & $\bullet$ & M & $\mathrm{EO}$ & próx. VII \\
\hline \multicolumn{5}{|l|}{ Scincidae } \\
\hline Mabuya frenata (Cope, 1862) & $\bullet$ & - & AIQ & $2 ; 4 ; 5 ; 6$ \\
\hline \multicolumn{5}{|l|}{ Teiidae } \\
\hline Ameiva ameiva (Linnaeus, 1758) & $\bullet$ & - & AIQ & $1 ; 5$ \\
\hline Cnemidophorus gr. ocellifer & $\bullet$ & - & AIQ & 1 \\
\hline Tupinambis merianae (Duméril \& Bibron, 1839) & $\bullet$ & - & AIQ; EO & $5 ; 6$ \\
\hline \multicolumn{5}{|l|}{ Anomalepididae } \\
\hline Liotyphlops beui Amaral, 1924 & - & I & - & - \\
\hline \multicolumn{5}{|l|}{ Boidae } \\
\hline Boa constrictor Linnaeus, 1758 & $\bullet$ & I & $\mathrm{EO} ; \mathrm{PV}$ & 4/IV \\
\hline Epicrates cenchria (Linnaeus, 1758) & - & I & - & - \\
\hline \multicolumn{5}{|l|}{ Colubridae } \\
\hline Chironius flavolineatus (Boettger, 1885) & - & I & - & - \\
\hline Chironius quadricarinatus (Boie, 1827) & - & I & - & - \\
\hline Mastigodryas bifossatus (Raddi, 1820) & - & I & - & - \\
\hline Simophis rhinostoma (Schlegel, 1837) & - & I & - & - \\
\hline \multicolumn{5}{|l|}{ Dipsadidae } \\
\hline Apostolepis dimidiata (Jan, 1862) & - & I & - & - \\
\hline Erythrolamprus aesculapii (Linnaeus, 1766) & $\bullet$ & I & AIQ & 1 \\
\hline Helicops carinicaudus (Wied, 1825) & - & I & - & - \\
\hline Helicops infrataeniatus (Jan, 1865) & - & I & - & - \\
\hline Hydrodynastes bicinctus (Herrmann, 1804) & - & I & - & - \\
\hline Imantodes cenchoa (Linnaeus, 1758) & - & I & - & - \\
\hline Leptodeira annulata (Linnaeus, 1758) & - & I & - & - \\
\hline Liophis frenatus (Werner, 1909) & - & I & - & - \\
\hline Liophis miliaris (Linnaeus, 1758) & - & I & - & - \\
\hline Liophis poecilogyrus (Wied, 1825) & - & I & - & - \\
\hline Liophis reginae (Linnaeus, 1758) & - & I & - & - \\
\hline Oxyrhopus guibei Hoge \& Romano, 1978 & $\bullet$ & I & AIQ; EO & 1 \\
\hline Oxyrhopus petola (Linnaeus, 1758) & $\bullet$ & I & PV & I \\
\hline Oxyrhopus rhombifer Duméril, Bibron \& Duméril, 1854 & - & I & - & - \\
\hline Oxyrhopus trigeminus Duméril, Bibron \& Duméril, 1854 & - & I & - & - \\
\hline Phalotris lativittatus Ferrarezzi, 1994 & $\bullet$ & & AIQ & 6 \\
\hline Phalotris mertensi (Hoge, 1955) & $\bullet$ & I & AIQ & 6 \\
\hline Philodryas mattogrossensis Koslowsky, 1898 & - & I & - & - \\
\hline Philodryas olfersii (Lichtenstein, 1823) & $\bullet$ & I & AIQ & 6 \\
\hline Philodryas patagoniensis (Girard, 1858) & $\bullet$ & $\mathrm{I} ; \mathrm{M}$ & $\mathrm{EO}$ & - \\
\hline Sibynomorphus mikanii (Schlegel, 1837) & - & I & - & - \\
\hline Taeniophallus occipitalis (Jan, 1863) & - & I & - & - \\
\hline Thamnodynastes nattereri (Mikan, 1828) & - & I & - & - \\
\hline Thamnodynastes rutilus (Prado, 1942) & - & I & - & - \\
\hline Xenodon merremii (Wagler, 1824) & - & I & - & - \\
\hline \multicolumn{5}{|l|}{ Elapidae } \\
\hline Micrurus frontalis (Duméril, Bibron \& Duméril, 1854) & - & $\mathrm{I} ; \mathrm{Z}$ & - & - \\
\hline Micrurus lemniscatus (Linnaeus, 1758) & $\bullet$ & I & $\mathrm{EO}$ & - \\
\hline \multicolumn{5}{|l|}{ Viperidae } \\
\hline Bothropoides jararaca (Wied, 1824) & $\bullet$ & I & AIQ; PV & 3 \\
\hline Bothropoides pauloensis (Amaral, 1925) & - & I & - & - \\
\hline Bothrops moojeni Hoge, 1966 & $\bullet$ & I & AIQ; PV & 6 \\
\hline Crotalus durissus (Laurenti, 1768) & $\bullet$ & $\mathrm{I}$ & AIQ; EO; PV & $2 ; 3 ; 4 / \mathrm{II}$ \\
\hline Rhinocerophis itapetiningae (Boulenger, 1907)1 & - & I & - & - \\
\hline
\end{tabular}

${ }^{1}$ Espécie vulnerável (VU) segundo a lista de espécies ameaçadas do estado de São Paulo (São Paulo 2008). 

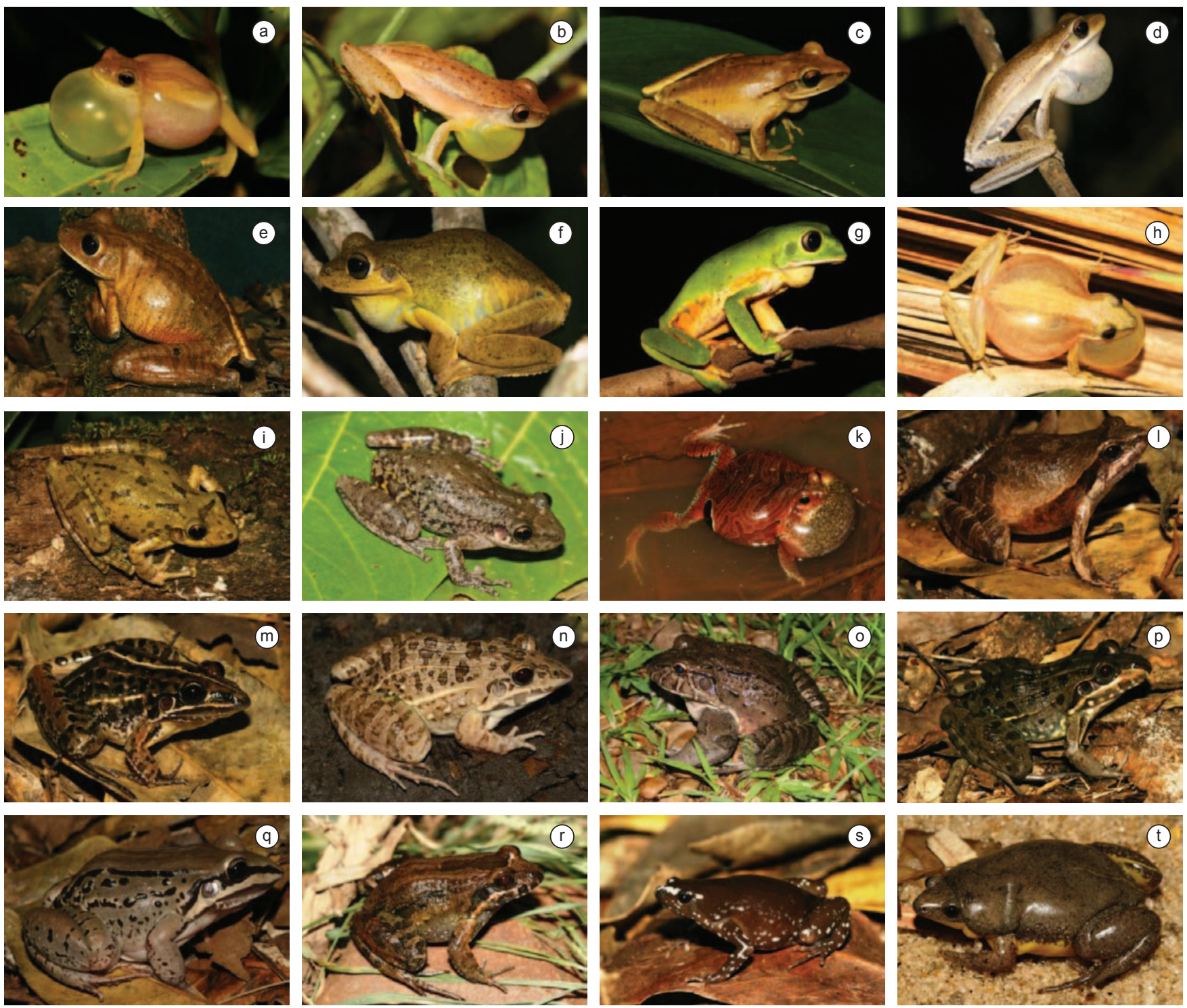

Figura 4. Espécies de anfíbios amostradas na Estação Ecológica de Assis, município de Assis, SP. a) Dendropsophus minutus; b) Dendropsophus nanus; c) Hypsiboas albopunctatus; d) Hypsiboas caingua; e) Hypsiboas faber; f) Hypsiboas lundii; g) Phyllomedusa tetraploidea; h) Scinax fuscomarginatus; i) Scinax fuscovarius; j) Scinax similis; k) Eupemphix nattereri; 1) Physalaemus cuvieri; m) Leptodactylus furnarius; n) Leptodactylus fuscus; o) Leptodactylus labyrinthicus; p) Leptodactylus latrans; q) Leptodactylus mystacinus; r) Leptodactylus podicipinus; s) Chiasmocleis albopunctata; t) Elachistocleis bicolor. Todos os indivíduos são da localidade estudada (fotos: Cybele O. Araujo).

Figure 4. Amphibian species sampled at the Estação Ecológica de Assis, municipality of Assis, state of São Paulo, Brazil. a) Dendropsophus minutus; b) Dendropsophus nanus; c) Hypsiboas albopunctatus; d) Hypsiboas caingua; e) Hypsiboas faber; f) Hypsiboas lundii; g) Phyllomedusa tetraploidea; h) Scinax fuscomarginatus; i) Scinax fuscovarius; j) Scinax similis; k) Eupemphix nattereri; 1) Physalaemus cuvieri; m) Leptodactylus furnarius; n) Leptodactylus fuscus; o) Leptodactylus labyrinthicus; p) Leptodactylus latrans; q) Leptodactylus mystacinus; r) Leptodactylus podicipinus; s) Chiasmocleis albopunctata; t) Elachistocleis bicolor. All individuals were found at the study site (photos: Cybele O. Araujo).

apresentaram uma tendência à estabilização, indicando que boa parte das espécies não foi registrada durante o estudo. Os estimadores apontam que podem ocorrer até 11 espécies a mais (riqueza observada $=17 \pm 2,53$; Bootstrap $=20,41$; Jacknife $\mathrm{I}=24,77 \pm 2,81$ ) Répteis são menos abundantes e mais difíceis de serem amostrados que anfíbios. Neste sentido, as curvas de rarefação demonstram que seria necessário maior esforço amostral para produzir uma lista mais completa para os répteis. A riqueza de répteis encontrada para o município de Assis (53 espécies), considerando dados deste estudo e registros de coleções científicas, indica que deve ocorrer na Estação
Ecológica de Assis um maior número de espécies, principalmente de serpentes.

\section{Espécies ameaçadas e endêmicas no estado de São Paulo}

Das espécies encontradas na Estação Ecológica de Assis e município de Assis, Micrablepharus atticolus e Rhinocerophis itapetiningae (vulnerável) estão presentes na lista de espécies ameaçadas de extinção para o estado de São Paulo (São Paulo 2008) (Tabela 3). Na Estação Ecológica de Assis, M. atticolus (Figura 5e) foi observada em área de cerrado típico (Tabelas 1 e 3). Outros estudos já demonstraram 

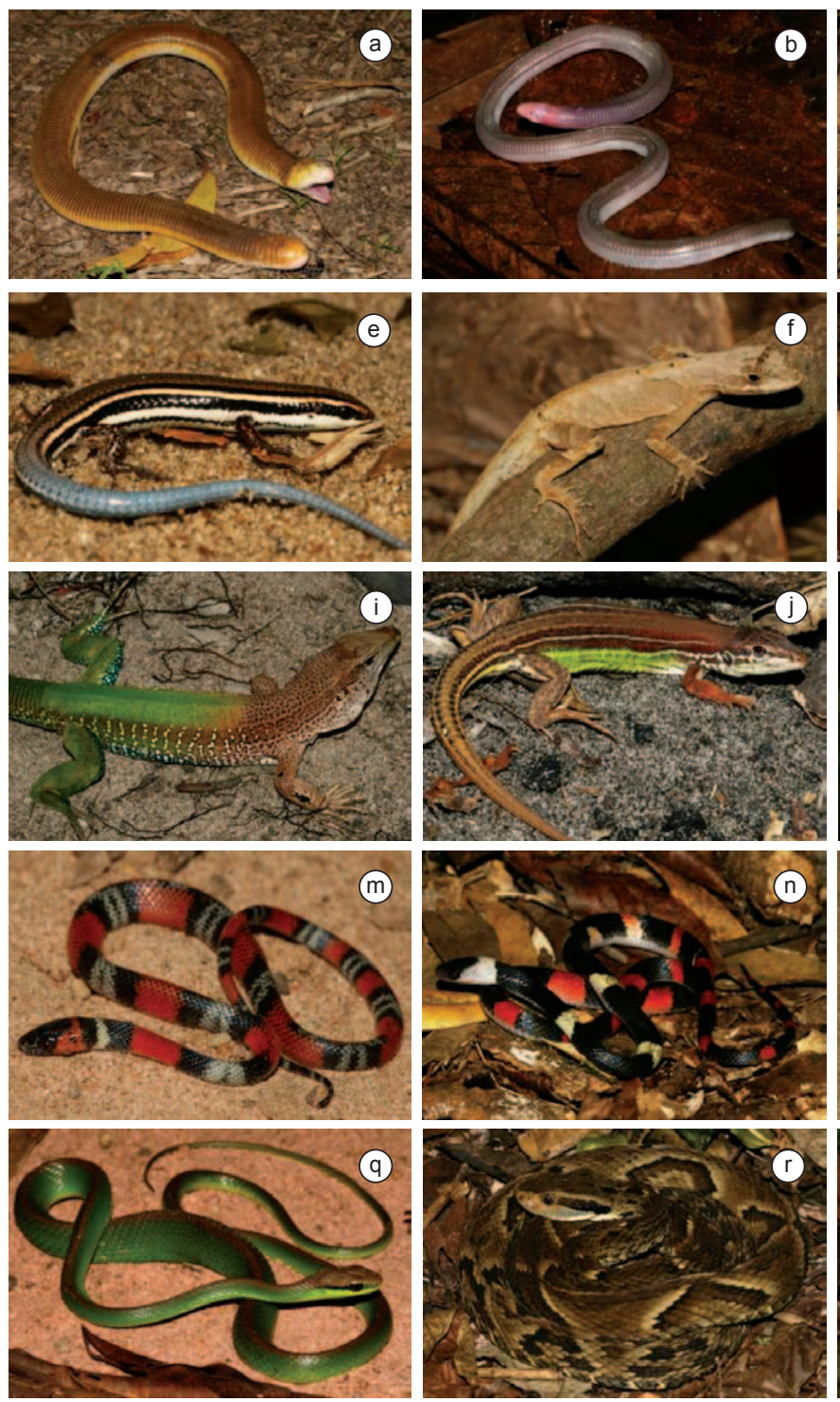
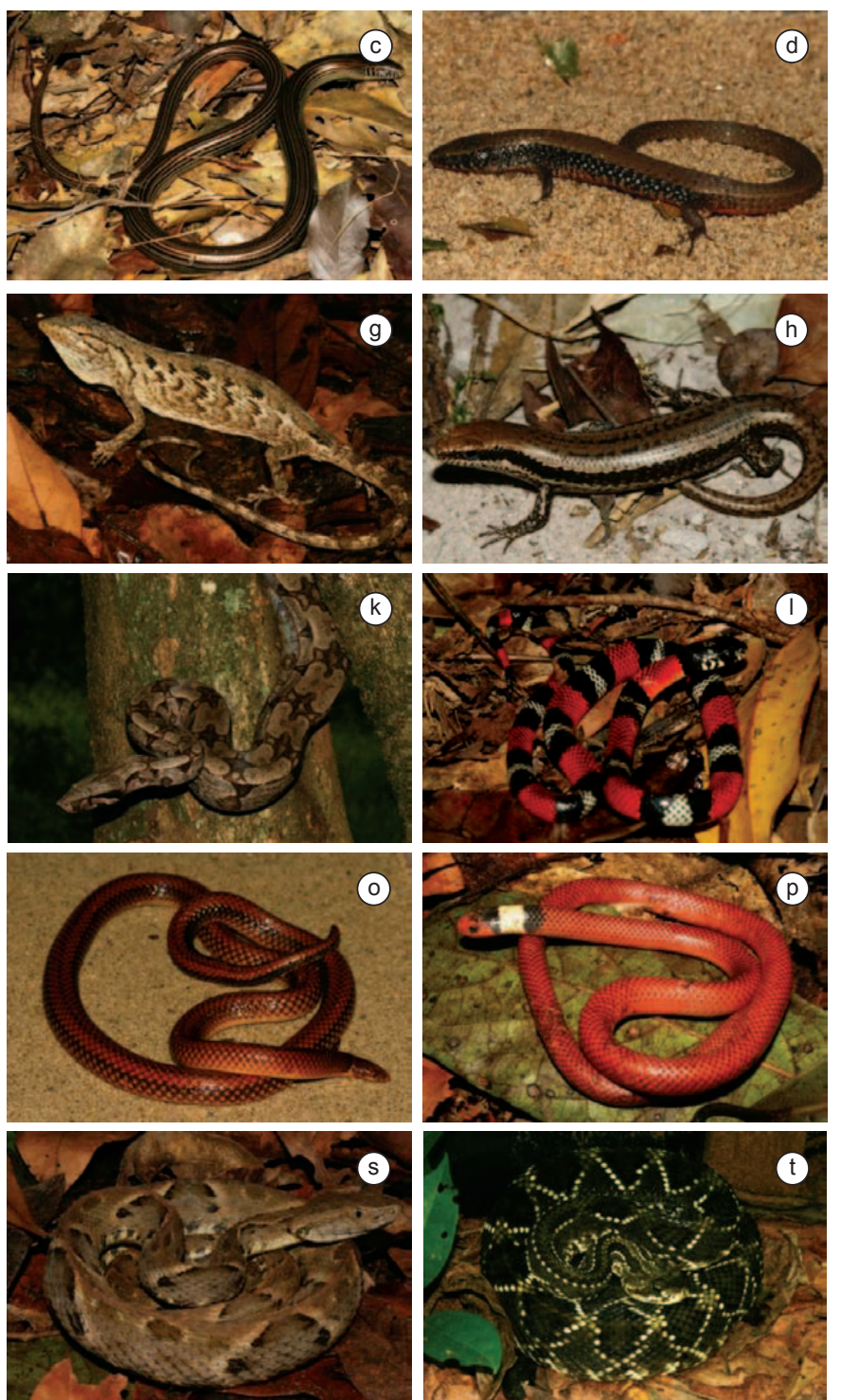

Figura 5. Espécies de répteis amostradas na Estação Ecológica de Assis, município de Assis, SP. a) Amphisbaena alba; b) Amphisbaena roberti; c) Ophiodes sp.; d) Colobosaura modesta; e) Micrablepharus atticolus; f) Anolis chrysolepis; g) Polychrus acutirostris; h) Mabuya frenata; i) Ameiva ameiva; j) Cnemidophorus gr. ocellifer; k) Boa constrictor; 1) Erythrolamprus aesculapii; m) Oxyrhopus guibei; n) Oxyrhopus petola; o) Phalotris lativittatus; p) Phalotris mertensi; q) Philodryas olfersii; r) Bothropoides jararaca; s) Bothrops moojeni; t) Crotalus durissus. Todos os indivíduos são da localidade estudada (fotos: Cybele O. Araujo).

Figure 5. Reptile species sampled at the Estação Ecológica de Assis, municipality of Assis, state of São Paulo, Brazil. a) Amphisbaena alba; b) Amphisbaena roberti; c) Ophiodes sp.; d) Colobosaura modesta; e) Micrablepharus atticolus; f) Anolis chrysolepis; g) Polychrus acutirostris; h) Mabuya frenata; i) Ameiva ameiva; j) Cnemidophorus gr. ocellifer; k) Boa constrictor; 1) Erythrolamprus aesculapii; m) Oxyrhopus guibei; n) Oxyrhopus petola; o) Phalotris lativittatus; p) Phalotris mertensi; q) Philodryas olfersii; r) Bothropoides jararaca; s) Bothrops moojeni; t) Crotalus durissus. All individuals were found at the study site (photos: Cybele O. Araujo).

que a ocorrência de $M$. atticolus (Nogueira 2006, Thomé 2006) e de $R$. itapetiningae (Marques et al. 2006, Sawaya et al. 2008) está restrita às fisionomias abertas de Cerrado, as formações vegetais mais vulneráveis ao desaparecimento no estado de São Paulo (Durigan et al. 2003, Durigan \& Ratter 2006) e na Estação Ecológica de Assis (Pinheiro 2008). Desta forma, para a efetiva conservação das populações locais destas espécies, é necessária a manutenção das características naturais da restrita área de cerrado típico $(0,6 \%)$ presente nesta unidade de conservação (Figura 2) e de outras áreas com formações campestres e savânicas presentes na região.
Dentre as espécies da herpetofauna registradas para o município de Assis (27 anfíbios e 53 répteis) apenas a serpente Phalotris lativittatus (Figura 5o) é endêmica do estado de São Paulo (Vasconcelos \& Santos 2009, Zaher et al. 2011). Esta espécie rara não havia sido registrada para o município de Assis antes deste estudo. Durante a amostragem, uma fêmea grávida foi capturada no cerradão em regeneração na Estação Ecológica de Assis. Informações sobre a história natural deste indivíduo como fecundidade, tamanho dos ovos, massa relativa da ninhada, período de incubação e tamanho dos jovens estão presentes em Braz et al. (2009). 

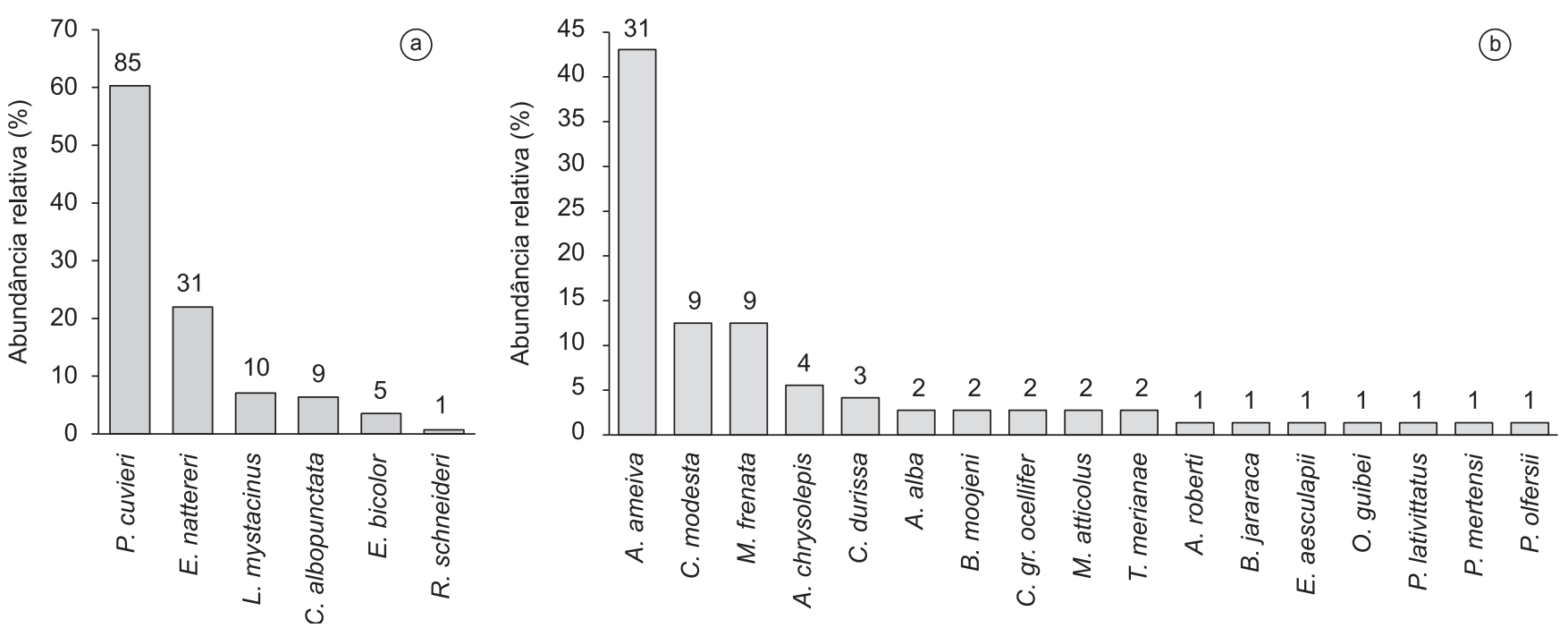

Figura 6. Abundância relativa das espécies de: a) anfíbios (número total de indivíduos $=141$ ); b) répteis (número total de indivíduos $=73$ ) amostradas por meio de armadilhas de interceptação e queda (AIQ) na Estação Ecológica de Assis, SP.

Figure 6. Relative abundance of: a) amphibians (total number of individuals = 141); b) reptiles (total number of individuals $=73$ ) species sampled by pitfall traps (AIQ) at the Estação Ecológica de Assis, state of São Paulo.

\section{Agradecimentos}

Somos gratas ao diretor e funcionários da Estação Ecológica de Assis pelo apoio logístico durante este estudo; a Décio T. Corrêa Filho, Edivaldo Furlan e Thais H. Condez pela grande ajuda nos trabalhos de campo; a Célio F. B. Haddad, Miguel T. Rodrigues e Valdir Germano pela contribuição na identificação dos espécimes; a Giselda Durigan pelo auxílio botânico; a Eduardo S. Pinheiro pela confecção dos mapas e ao Instituto Florestal pelo suporte financeiro. Selma M. Almeida-Santos agradece ao CNPq e a FAPESP pelo apoio financeiro.

\section{Referências Bibliográficas}

AB'SÁBER, A.N. 2005. Os domínios de natureza no Brasil: potencialidades paisagísticas. Ateliê Editorial, São Paulo.

AGUIAR JUNIOR, O., BACCI JUNIOR, M., LIMA, A.P., ROSSAFERES, D.C., HADDAD, C.F.B. \& RECCO-PIMENTEL, S.M. 2007. Phylogenetic relationships of Pseudis and Lysapsus (Anura, Hylidae, Hylinae) inferred from mitochondrial and nuclear gene sequences. Cladistics 23:1-9.

ARAUJO, C.O., CONDEZ, T.H. \& SAWAYA, R.J. 2009a. Anfíbios anuros do Parque Estadual das Furnas do Bom Jesus, sudeste do Brasil, e suas relações com outras taxocenoses no Brasil. Biota Neotrop. 9(2):77-98, http://www. biotaneotropica.org.br/v9n2/pt/abstract?article+bn01109022009 (último acesso em 13/02/2010).

ARAUJO, C.O., CONDEZ, T.H., BOVO, R.P., CENTENO, F.C. \& LUIZ, A.M. 2010a. Amphibians and reptiles of the Parque Estadual Turístico do Alto Ribeira (PETAR), SP: an Atlantic Forest remnant of southeastern Brazil. Biota Neotrop. 10(4): http://www.biotaneotropica.org.br/v10n4/ pt/abstract?inventory+bn01710042010 (último acesso em 02/03/2011).

ARAUJO, C.O., CORRÊA-FILHO, D.T. \& SAWAYA, R.J. 2010b. Snake assemblage of Estação Ecológica de Santa Bárbara, SP: a Cerrado remnant in Southeastern Brazil. Biota Neotrop. 10(2): http://www.biotaneotropica. org.br/v10n2/pt/abstract?inventory+bn01410022010 (último acesso em 05/11/2010).

ARAÚJO, O.G.S., TOLEDO, L.F., GARCIA, P.C.A. \& HADDAD, C.F.B. 2009b. The amphibians of São Paulo State. Biota Neotrop. 9(4): http://www.biotaneotropica.org.br/v9n4/en/abstract?inventory+ bn03109042009 (último acesso em 20/04/2010).
BÉRNILS, R.S. 2010. Répteis brasileiros - Lista de espécies. Sociedade Brasileira de Herpetologia. Disponível em http://www.sbherpetologia. org.br/ (último acesso em 07/03/2011).

BISBY, F.A., ROSKOV, Y.R., ORRELL, T.M., NICOLSON, D., PAGLINAWAN, L.E., BAILLY, N., KIRK, P.M., BOURGOIN, T. \& BAILLARGEON, G. 2010. Species 2000 \& ITIS Catalogue of Life: 2010 Annual Checklist. Disponível em http://www.catalogueoflife.org/ annual-checklist/2010 (último acesso em 09/11/2010).

BRANDÃO, R.A. \& ARAÚJO, A.F.B. 2002. A herpetofauna associada a matas de galeria no Distrito Federal. In Cerrado: caracterização e recuperação de matas de galeria (J.F. Ribeiro, C.E.L. Fonseca \& J.C. Sousa-Silva, orgs.). EMBRAPA/CPAC, Planaltina, p.560-604.

BRASIL. Ministério do Meio Ambiente - MMA \& INSTITUTO BRASILEIRO DO MEIO AMBIENTE E DOS RECURSOS NATURAIS RENOVÁVEIS - IBAMA. 2009. Relatório técnico de monitoramento do desmatamento no Bioma Cerrado, 2002 a 2008: dados revisados. Disponível em http://siscom.ibama.gov.br/monitorabiomas/cerrado/ Relatoriotecnico_MonitoramentoDesmate_BiomaCerrado_CSR_REV. pdf (último acesso em: 23/12/2010).

BRASILEIRO, C.A. 2004. Diversidade de anfíbios anuros em área de Cerrado no estado de São Paulo. Tese de doutorado, Universidade de São Paulo, São Paulo.

BRASILEIRO, C.A., SAWAYA, R.J., KIEFER, M.C. \& MARTINS, M. 2005. Amphibians of an open Cerrado fragment in southeastern Brazil. Biota Neotrop. 5(2): http://www.biotaneotropica.org.br/v5n2/pt/ abstract?article+BN00405022005 (último acesso em 16/07/2010).

BRASSALOTI, R.A., ROSSA-FERES, D.C. \& BERTOLUCI, J. 2010. Anurofauna da Floresta Estacional Semidecidual da Estação Ecológica dos Caetetus, sudeste do Brasil. Biota Neotrop. 10(1): http://www. biotaneotropica.org.br/v10n1/pt/abstract?inventory+bn01810012010 (último acesso em 16/07/2010).

BRAZ, H.B.P., ARAUJO, C.O. \& ALMEIDA-SANTOS, S.M. 2009. Life history traits of the snake Phalotris lativittatus (Xenodontinae: Elapomorphini) from the Brazilian Cerrado. Herpetology Notes 2:163164.

CECHIN, S.Z. \& MARTINS, M. 2000. Eficiência de armadilhas de queda (Pitfall traps) em amostragens de anfíbios e répteis no Brasil. Rev. Bras. Zool. 17(3):729-749. http://dx.doi.org/10.1590/S010181752000000300017 
CHAPARRO, J.C., PRAMUK, J.B. \& GLUESENKAMP, A.G. 2007 A new species of arboreal Rhinella (Anura: Bufonidae) from cloud forest of southeastern Peru. Herpetologica 63:203-212. http://dx.doi. org/10.1655/0018-0831(2007)63[203:ANSOAR]2.0.CO;2

CICCHI, P.J.P., SENA, M.A., PECCININI-SEALE, D.M. \& DUARTE, M.R. 2007. Serpentes das ilhas costeiras do Estado de São Paulo, Sudeste do Brasil. Biota Neotrop. 7(2): http://www.biotaneotropica.org.br/v7n2/pt/ abstract?inventory+bn03907022007 (último acesso em: 16/07/2010).

COLLI, G.R., BASTOS, R.P. \& ARAÚJO, A.F.B. 2002. The character and dynamics of the Cerrado herpetofauna. In The cerrados of Brazil: ecology and natural history of a Neotropical Savanna (P.S. Oliveira \& R.J. Marquis, eds.). Columbia University Press, New York, p.223-241.

COLWELL, R.K. 2009. EstimateS: statistical estimation of species richness and shared species from samples. Versão 8. 2. 0. Disponível em: http:// viceroy.eeb.uconn.edu/estimates (último acesso em: 10/07/2010).

CONDEZ, T.H., SAWAYA, R.J. \& DIXO, M. 2009. Herpetofauna dos remanescentes de Mata Atlântica da região de Tapiraí e Piedade, SP, sudeste do Brasil. Biota Neotrop. 9(1): http://www.biotaneotropica. org.br/v9n1/en/abstract?inventory+bn01809012009. (último acesso em 20/04/2010)

CORN, P.S. 1994. Straight-line drift-fences and pitfall traps. In Measuring and monitoring biological diversity. Standard methods for amphibians (W.R. Heyer, M.A. Donnely, R.W. McDiarmid, L.C. Hayek \& M.S. Foster, eds.). Smithsonian institution Press, Washington and London, p.109-117.

CRUMP, M.L. \& SCOTT JUNIOR, N.J. 1994. Standard techniques for inventory and monitoring. In Measuring and monitoring biological diversity: standard methods for amphibians (W.R. Heyer, M.A. Donnelly, R.W. Mc Diarmid, L.C. Hayek \& M.S Foster, eds.). Smithsonian Institution Press, Washington and London, p.84-92.

CURCIO, F.F., PIACENTINI, V.Q. \& FERNANDES, D.S. 2009. On the status of the snake genera Erythrolamprus Boie, Liophis Wagler and Lygophis Fitzinger (Serpentes, Xenodontinae). Zootaxa. 2173:66-68.

DALMOLIN, P.C. 2000. Composição e história natural da comunidade de serpentes da Estação Ecológica de Jataí e outras áreas do município de Luiz Antonio, (SP). Dissertação de Mestrado, Universidade Federal de São Carlos, São Carlos.

DURIGAN, G. 2010. Plano de Manejo da Estação Ecológica de Assis. Instituto Florestal, Secretaria do Meio Ambiente do estado de São Paulo, São Paulo.

DURIGAN, G., FRANCO, G.A.D.C. \& SIQUEIRA, M.F. 2004. A vegetação dos remanescentes de cerrado no Estado de São Paulo. In Viabilidade da conservação dos remanescentes de Cerrado no Estado de São Paulo (M.D. Bitencourt \& R.R. Mendonça, orgs.). Annablume, FAPESP, São Paulo, p.29-56.

DURIGAN, G. \& PINHEIRO, E.S. 2010. Vegetação. In Plano de Manejo da Estação Ecológica de Assis (G. Durigan, org.). Instituto Florestal, Secretaria do Meio Ambiente do estado de São Paulo, São Paulo, p.51-58.

DURIGAN, G. \& RATTER, J.A. 2006. Successional changes in Cerrado and Cerrado/forest ecotonal vegetation in Western São Paulo State, Brazil, 1962-2000. Edinburgh J. Bot. 63(1):119-130. http://dx.doi.org/10.1017/ S0960428606000357

DURIGAN, G., SIQUEIRA, M.F. \& FRANCO, G.A.D.C. 2007. Threats to the Cerrado remnants of the State of São Paulo, Brazil. Braz. Sci. Agric. 64(4):355-363.

DURIGAN, G., SIQUEIRA, M.F., FRANCO, G.A.D.C., BRIDGEWATER, S. \& RATTER, J.A. 2003. The vegetation of priority areas for cerrado conservation in São Paulo State, Brazil. Edinburgh J. Bot. 60(2):217-241.

FAIVOVICH, J., HADDAD, C.F.B., GARCIA, P.C.A., FROST, D.R., CAMPBELL, J.A. \& WHEELER, W.C. 2005. Systematic review of the frog family Hylidae, with special reference to Hylinae: a phylogenetic analysis and taxonomic revision. Bull. Am. Mus. Nat. Hist. 294:1-240. http://dx.doi.org/10.1206/0003-0090(2005)294[0001:SROTFF]2.0.CO;2
FENWICK, A.M., GUTBERLET JUNIOR, R.L., EVANS, J.A. \& PARKINSON, C.L. 2009. Morphological and molecular evidence for phylogeny and classification of South American pitvipers, genera Bothrops, Bothriopsis, and Bothrocophias (Serpentes: Viperidae). Zool. J. Lin. Soc. 156:617-640. http://dx.doi.org/10.1111/j.10963642.2008.00495

FONSECA, G.A.B., CAVALCANTI, R.B., RYLANDS, A.B. \& PAGLIA, A.P. 2004. Cerrado. In Hotspots revisited: earth's biologically richest and most endangered terrestrial ecoregions (R.A. Mittermeier, P.R. Gil, M. Hoffman, J. Pilgrim, T. Brooks, C.G. Mittermeier, J. Lamoreux \& G.A.B. Fonseca, eds.). CEMEX \& Agrupacion Sierra Madre, Cidade do México, p.93-97.

FONSECA, G.A.B., MITTERMEIER, R.A., CAVALCANTI, R.B. \& MITTERMEIER, C.G. 1999. Brazilian Cerrado. In Hotspots: earth's biologically richest and most endangered terrestrial (R.A. Mittermeier, N. Myers, P.R. Gil \& C.G. Mittermeier, eds.). CEMEX \& Agrupacion Sierra Madre, Cidade do México, p.148-159.

FROST, D.R. 2011. Amphibian species of the world: an online reference. Version 5.5. Electronic Database. American Museum of Natural History, New York. Disponível em http://research.amnh.org/vz/herpetology/ amphibia (último acesso em 07/03/2011).

FROST, D.R., ETHERIDGE, R.E., JANIES, D. \& TITUS, T.A. 2001. Total evidence, sequence alignment, evolution of polychrotid lizards, and a reclassification of the Iguania (Squamata: Iguania). Am. Mus. Novit. 3343:1-38. http://dx.doi.org/10.1206/0003-0082(2001)343\%3C0001:T ESAEO\%3E2.0.CO;2

FROST, F.D., GRANT, T., FAIVOVICH, J., BAIN, R.H., HAAS, A., HADDAD, C.F.B., DE SÁ, R.O., CHANNING, A., WILKINSON, M., DONNELLAN, S.C., RAXWORTHY, C.J., CAMPBELL, J.A., BLOTTO, B.L., MOLER, P., DREWES, R.C., NUSSBAUM, R.A., LYNCH, J.D., GREEN, D.M. \& WHEELER, W.C. 2006. The Amphibian Tree of Life. Bull. Am. Mus. Nat. Hist. 297:1-370. http://dx.doi. org/10.1206/0003-0090(2006)297[0001:TATOL]2.0.CO;2

GOTELLI, N.J. \& COLWELL, R.K. 2001. Quantifying biodiversity: procedures and pitfalls in the measurement and comparison of species richness. Ecol. Lett. 4(4):379-391. http://dx.doi.org/10.1046/j.14610248.2001 .00230

GRANT, T., FROST, D.R., CALDWELL, J.P., GAGLIARDO, R., HADDAD, C.F.B., KOK, P.J.R., MEANS, D.B., NOONAN, B.P., SCHARGEL, W.E. \& WHEELER, W.C. 2006. Phylogenetic systematics of dart-poison frogs and their relatives (Amphibia: Athesphatanura: Dendrobatidae) Bull. Am. Mus. Nat. Hist. 299:1-262. http://dx.doi.org/10.1206/00030090(2006)299[1:PSODFA]2.0.CO;2

HEYER, W.R., RAND, A.S., CRUZ, C.A.G., PEIXOTO, O.L. \& NELSON, C.E. 1990. Frogs of Boracéia. Arq. Zool. 31(4):231-410.

INSTITUTO BRASILEIRO DE GEOGRAFIA E ESTATÍSTICA - IBGE. 2004. Mapa de Biomas do Brasil: primeira aproximação. IBGE, Rio de Janeiro.

INSTITUTO BRASILEIRO DO MEIO AMBIENTE E DOS RECURSOS NATURAIS RENOVÁVEIS - IBAMA. 2003. Lista nacional das espécies da fauna brasileira ameaçadas de extinção. IBAMA. Disponível em http:// www.mma.gov.br/sitio/index.php?ido=conteudo.monta\&idEstrutura $=179$ (último acesso em: 18/05/2010).

INTERNATIONAL UNION FOR CONSERVATION OF NATURE - IUCN. 2010. The IUCN red list of threatened species. Version 2010.4. Disponível em http://www.iucnredlist.org (último acesso em: 07/03/2011).

JIM, J. 1980. Aspectos ecológicos dos anfíbios registrados na região de Botucatu, São Paulo (Amphibia, Anura). Tese de doutorado, Universidade de São Paulo, São Paulo.

KLINK, C.A. \& MACHADO, R.B. 2005. Conservation of the Brazilian Cerrado. Conserv. Biol. 19(3):707-713. http://dx.doi.org/10.1111/j.15231739.2005.00702.x

KRONKA, F.J.N., NALON, M.A. \& MATSUKUMA, C.K. 2005. Inventário florestal da vegetação natural do estado de São Paulo. Secretaria do Meio Ambiente, Instituto Florestal, Imprensa Oficial, São Paulo. 
LAVILLA, E.O., LANGONE, J.A., CARAMASCHI, U., HEYER, W.R. \& DE SÁ, R.O. 2010. The identification of Rana ocellata Linnaeus, 1758. Nomenclatural impact on the species currently known as Leptodactylus ocellatus (Leptodactylidae) and Osteopilus brunneus (Gosse, 1851) (Hylidae). Zootaxa. 2346:1-16.

MACHADO, R.B., RAMOS NETO, M.B., PEREIRA, P.G.P., CALDAS, E.F., GONÇALVES, D.A., SANTOS, N.S., TABOR, K. \& STEININGER, M. 2004. Estimativas de perda da área do Cerrado brasileiro. Conservation International do Brasil, Brasília.

MALAGOLI, L.R. 2008. Anfíbios do município de São Paulo: histórico, conhecimento atual e desafios para a conservação. In Além do concreto: contribuições para a proteção da biodiversidade paulistana (L.R. Malagoli, F.B. Bajesteiro \& M. Whately, orgs.). Instituto Sócio Ambiental, São Paulo, p.204-233.

MARQUES, O.A.V., ETEROVIC, P.C. \& SAZIMA, I. 2004. Snakes of the Brazilian Atlantic forest: an illustrated field guide for the Serra do Mar Range. Ed. Holos, Ribeirão Preto.

MARQUES, O.A.V., PEREIRA, D.N., BARBO, F.E., GERMANO, V.J. \& SAWAYA, R.J. 2009. Os Répteis do Município de São Paulo: diversidade e ecologia da fauna pretérita e atual. Biota Neotrop. 9(2):139-150, http:// www.biotaneotropica.org.br/v9n2/pt/abstract?article+bn02309022009 (último acesso em 18/06/2010).

MARQUES, O.A.V., SAWAYA, R.J., STENDER-OLIVEIRA, F. \& FRANÇA, F.G.R. 2006. Ecology of the colubrid snake Pseudablabes agassizii in southeastern South America. Herpetol. J. 16(1):37-45.

MARTINS, M. 1994. História Natural e Ecologia de uma taxocenose de serpentes em Mata Atlântica Primária na região de Manaus, Amazônia Central, Brasil. Tese de Doutorado, Universidade Estadual de Campinas, Campinas.

MITTERMEIER, R.A., GIL, P.R., HOFFMAN, M., PILGRIM, J., BROOKS, T., MITTERMEIER, C.G., LAMOREUX, J. \& FONSECA, G.A.B. 2004. Hotspots revisited: earth's biologically richest and most endangered terrestrial ecoregions. CEMEX \& Agrupacion Sierra Madre, Cidade do México.

MORAES, R.A., SAWAYA, R.J. \& BARRELA, W. 2007. Composição e diversidade de anfíbios anuros em dois ambientes de Mata Atlântica no Parque Estadual Carlos Botelho, São Paulo, sudeste do Brasil. Biota Neotrop. 7(2): http://www.biotaneotropica.org.br/v7n2/pt/ abstract?article+bn00307022007 (último acesso em 16/07/2010).

MOTT, T. \& VIEITES, D.R. 2009. Molecular phylogenetics reveals extreme morphological homoplasy in Brazilian worm lizards challenging current taxonomy. Mol. Phylogenet. Evol. 51(2):190-200. http://dx.doi. org/10.1016/j.ympev.2009.01.014

MYERS, N., MITTERMEIER, R.A., MITTERMEIER, C.G., FONSECA, G.A.B. \& KENT, J. 2000. Biodiversity hotspots for conservation priorities. Nature 403:853-858. http://dx.doi.org/10.1038/35002501

NARVAES, P., BERTOLUCI, J.A. \& RODRIGUES, M.T. 2009. Composição, uso de hábitat e estações reprodutivas das espécies de anuros da floresta de restinga da Estação Ecológica Juréia-Itatins, sudeste do Brasil. Biota Neotrop. 9(2): http://www.biotaneotropica.org.br/v9n2/ pt/abstract?article+bn02009022009 (último acesso em: 16/07/2010).

NASCIMENTO, L.B., PIMENTA, B.V.S., CRUZ, C.A.G. \& CARAMASCHI, U. 2006. Taxonomic status of Gomphobates marmoratus Reinhardt and Lütken, 1862 "1861" and Eupemphix fuscomaculatus Steindachner, 1864 (Amphibia, Anura, Leptodactylidae. S. Amer. J. Herpetol. 1(3):166-174. http://dx.doi.org/10.2994/1808-9798(2006)1[166:TSOGMR]2.0.CO;2

NOGUEIRA, C.C. 2006. Diversidade e padrões de distribuição da fauna de lagartos do Cerrado. Tese de Doutorado, Universidade de São Paulo, São Paulo.
NOGUEIRA, C., COLLI, G.R. \& MARTINS, M. 2009. Local richness and distribution of the lizard fauna in natural habitat mosaics of the Brazilian Cerrado. Austral Ecol. 34:83-96. http://dx.doi.org/10.1111/j.14429993.2008.01887.x

PEEL, M.C., FINLAYSON, B.L. \& McMAHON, T.A. 2007. Updated world map of the Köppen-Geiger climate classification. Hydrol. Earth Syst. Sci. 11:1633-1644. http://dx.doi.org/10.5194/hess-11-1633-2007

PINHEIRO, E.S. 2008. Análises ecológicas e sensoriamento remoto aplicados a estimativa de fitomassa de Cerrado na Estação Ecológica de Assis, SP. Tese de Doutorado, Escola de Engenharia de São Carlos, Universidade de São Paulo, São Paulo.

PONÇANO, W.L., ALMEIDA, F.F.A., CARNEIRO, C.D.R., BISTRICHI, C.A., PIRES-NETO, A.C., ALMEIDA, M.A., PRANDINI, F.L., ÁVILA, I.G., FORNASARI-FILHO, N., IWASA, O.Y. \& SANTOS, M. 1981. Mapa geomorfológico do Estado de São Paulo. Instituto de Pesquisas Tecnológicas, São Paulo.

PRADO, V.H.M., SILVA, F.R., DIAS, N.Y.N., PIRES, J.S.R. \& ROSSAFERES, D.C. 2009. Anura, Estação Ecológica de Jataí, São Paulo State, southeastern Brazil. Check List 5(3):495-502.

RATTER, J.A., RIBEIRO, J.F. \& BRIDGEWATER, S. 1997. The Brazilian Cerrado vegetation and threats to its biodiversity. Ann. Bot. 80:223-230.

RIBEIRO, J.F. \& WALTER, B.M.T. 2008. As principais fitofisionomias do Bioma Cerrado. In Cerrado: ecologia e flora (S.M. Sano, S.P. Almeida \& J.F. Ribeiro, eds.). EMBRAPA, Planaltina, p.153-212.

RIBEIRO-JÚNIOR, J.W. \& BERTOLUCI, J. 2009. Anuros do cerrado da Estação Ecológica e da Floresta Estadual de Assis, sudeste do Brasil. Biota Neotrop. 9(1): http://www.biotaneotropica.org.br/v9n1/pt/abstrac t?inventory+bn02709012009 (último acesso em 16/07/2010).

ROSSA-FERES, D.C. \& JIM, J. 1994. Distribuição sazonal em comunidades de anfíbios anuros na região de Botucatu, São Paulo. Rev. Bras. Biol. 54(2):323-334.

ROSSA-FERES, D.C., SAWAYA, R.J., FAIVOVICH, J., GIOVANELLI, J.G.R., BRASILEIRO, C.A., SCHIESARI, L., ALEXANDRINO, J. \& HADDAD, C.F.B. 2011. Anfíbios do Estado de São Paulo, Brasil: Conhecimento Atual e Perspectivas. Biota Neotrop. 11(1a): http://www. biotaneotropica.org.br/v11n1a/pt/abstract?inventory+bn0041101a2011 (último acesso em 22/12/2010).

SÃO PAULO (Estado). 1997. Cerrado: Bases para a conservação e uso sustentável das áreas de Cerrado do Estado de São Paulo. SEMA, São Paulo. (Série PROBIO/SP).

SÃO PAULO (Estado). 2008. Decreto Estadual n ${ }^{\circ} 53.494$, de 02 de outubro de 2008. Diário Oficial do Estado de São Paulo, São Paulo, 03 out. 2008. Disponível em http://www.imprensaoficial.com.br (último acesso em 27/07/2010).

SAWAYA, R.J. 2004. História natural e ecologia das serpentes de Cerrado da região de Itirapina, SP. Tese de Doutorado, Universidade Estadual de Campinas, Campinas.

SAWAYA, R.J., MARQUES, O.A.V. \& MARTINS, M. 2008. Composition and natural history of a Cerrado snake assemblage at Itirapina, São Paulo State, southeastern Brazil. Biota Neotrop. 8(2): http://www.biotaneotropica. org.br/v8n2/en/abstract?inventory+bn01308022008 (último acesso em 16/07/2010).

SOCIEDADE BRASILEIRA DE HERPETOLOGIA - SBH. 2010. Brazilian amphibians - List of species. Disponível em http://www.sbherpetologia. org.br (último acesso em 07/03/2011).

THOMÉ, M.T.C. 2006. Diversidade de anuros e lagartos em fisionomias de Cerrado na região de Itirapina, sudeste do Brasil. Dissertação de Mestrado, Universidade de São Paulo, São Paulo.

TOLEDO, L.F., LOEBMANN, D. \& HADDAD, C.F.B. 2010. Revalidation and redescription of Elachistocleis cesarii (Miranda-Ribeiro, 1920) (Anura: Microhylidae). Zootaxa. 2418:50-60. 
VANZOLINI, P.E. 1948. Notas sobre os ofídios e lagartos da Cachoeira de Emas, no município de Pirassununga, Estado de São Paulo. Rev. Bras. Biol. 8:377-400.

VASCONCELOS, T.S. \& ROSSA-FERES, D.C. 2005. Diversidade, distribuição espacial e temporal de anfíbios anuros (Amphibia, Anura) na região noroeste do estado de São Paulo. Biota Neotrop. 5(2): http:// www.biotaneotropica.org.br/v5n2/pt/abstract?article+BN01705022005 (último acesso em 16/07/2010).

VASCONCELOS, T.S. \& SANTOS, T.G. 2009. Reptilia, Serpentes, Dipsadidae, Phalotris lativittatus: Distribution extension and geographic distribution map. Check List. 5(1):5-7.
ZAHER, H., BARBO, F.E., MARTÍNEZ, P.S., NOGUEIRA, C., RODRIGUES, M.T. \& SAWAYA R.J. 2011. Répteis do Estado de São Paulo: Conhecimento Atual e Perspectivas. Biota Neotrop. 11(1a): http:// www.biotaneotropica.org.br/v11n1a/pt/abstract?inventory+bn005110 1 a2011 (último acesso em 22/12/2010).

ZAHER, H., GRAZZIOTIN, F.G., CADLE, J.E., MURPHY, R.W., MOURALEITE, J.C. \& BONATTO, S.L. 2009. Molecular Phylogeny of advanced snakes (Serpentes, Caenophidia) with an emphasis on South American Xenodontines: A revised classification and descriptions of new taxa. Pap. Av. Zool. 49(11):115-153. http://dx.doi.org/10.1590/S003110492009001100001

Recebido em 10/11/2010 Versão reformulada recebida em 08/03/2011 Publicado em 07/07/2011 
Apêndice 1. Anfíbios e répteis coletados na Estação Ecológica de Assis, município de Assis, SP e depositados na Coleção de Anfíbios CFBH, Departamento de Zoologia, Instituto de Biociências, Universidade Estadual Paulista, Rio Claro, Coleção Herpetológica "Alphonse Richard Hoge", Instituto Butantan (IBSP and CRIB), São Paulo e Coleção Herpetológica do Museu de Zoologia da Universidade de São Paulo, São Paulo (MZUSP).

Chiasmocleis albopunctata (CFBH 18853, 18854, 20032-20034); Dendropsophus minutus (CFBH 20022-20024); Dendropsophus nanus (CFBH 18857-18859, 20038, 20039, 20053); Elachistocleis bicolor (CFBH 18851, 18852, 20035-20037); Eupemphix nattereri (CFBH 1883518837, 20018-20021); Hypsiboas albopunctatus (CFBH 18840, 18842, 18843, 20025-20027); Hypsiboas caingua (CFBH 18691-18693, 20041, 20042); Hypsiboas faber (CFBH 18830, 18831, 20058); Hypsiboas lundii (CFBH 18832, 18833, 20059); Leptodactylus furnarius (CFBH 18694, 20055); Leptodactylus fuscus (CFBH 18839, 20054); Leptodactylus labyrinthicus (CFBH 18845-18847, 19748, 20048, 20049); Leptodactylus latrans (CFBH 18698, 18699, 20057); Leptodactylus mystacinus (CFBH 18834, 18860, 20060, 20061); Leptodactylus podicipinus (CFBH 18844, 20056); Phyllomedusa tetraploidea (CFBH 18829, 20062, 20063); Physalaemus cuvieri (CFBH 18848-18850, 18861, 20043-20047); Physalaemus marmoratus (CFBH 18838, 20030, 20031); Rhinella schneideri (CFBH 18862); Scinax fuscomarginatus (CFBH 18855, 18856, 20040, 20051); Scinax fuscovarius (CFBH 18841, 20028, 20029); Scinax similis (CFBH 18695-18697, 20050, 20052); Ameiva ameiva (MZUSP 100083-100090); Amphisbaena alba (MZUSP 100091, 100092); Amphisbaena roberti (MZUSP 100093); Anolis chrysolepis (CRIB 0696-0698); Bothropoides jararaca (IBSP 76921); Bothrops moojeni (IBSP 76927, 76928); Cnemidophorus gr. ocellifer (MZUSP 100094, 100095); Colobosaura modesta (MZUSP 100096-100102); Crotalus durissus (IBSP 76922); Erythrolamprus aesculapii (IBSP 76920); Mabuya frenata (MZUSP 100103-100108); Micrablepharus atticolus (MZUSP 100109, 100110); Ophiodes sp. (MZUSP 100113); Oxyrhopus guibei (IBSP 76923, 76929); Oxyrhopus petola (IBSP 76924); Phalotris lativittatus (IBSP 76925); Phalotris mertensi (IBSP 76926); Philodryas olfersii (IBSP 76930); Polychrus acutirostris (MZUSP 100111, 100112). 